\title{
Permutations with Ascending and Descending Blocks
}

\author{
Jacob Steinhardt \\ jacob.steinhardt@gmail.com \\ Submitted: Aug 29, 2009; Accepted: Jan 4, 2010; Published: Jan 14, 2010 \\ Mathematics Subject Classification: 05A05
}

\begin{abstract}
We investigate permutations in terms of their cycle structure and descent set. To do this, we generalize the classical bijection of Gessel and Reutenauer to deal with permutations that have some ascending and some descending blocks. We then provide the first bijective proofs of some known results. We also extend the work done in [4] by Eriksen, Freij, and Wästlund, who study derangements that descend in blocks of prescribed lengths. In particular, we solve some problems posed in [4] and also obtain a new combinatorial sum for counting derangements with ascending and descending blocks.
\end{abstract}

\section{Introduction}

We consider permutations in terms of their descent set and conjugacy class (equivalently, cycle structure). Let $\pi$ be a permutation on $\{1, \ldots, n\}$. An ascent of $\pi$ is an index $i$, $1 \leqslant i<n$, such that $\pi(i)<\pi(i+1)$. A descent of $\pi$ is such an index with $\pi(i)>\pi(i+1)$.

The study of permutations by descent set and cycle structure goes back at least as far as 1993, when Gessel and Reutenauer enumerated them using symmetric functions [5]. In their proof, they obtained a bijection from permutations with at most a given descent set to multisets of necklaces with certain properties. By a necklace we mean a directed cycle where the vertices are usually assigned colors or numbers. Multisets of necklaces are usually referred to as ornaments. Figure 1 illustrates these terms.

The Gessel-Reutenauer bijection preserves cycle structure. It also forgets other structure that is not so relevant, making it easier to study permutations by cycle structure and descent set. We will restate Gessel's and Reutenauer's result to bring it closer to the language of more recent work $([6],[4])$. Choose $a_{1}, \ldots, a_{k}$ with $a_{1}+\cdots+a_{k}=n$, and partition $\{1, \ldots, n\}$ into consecutive blocks $A_{1}, \ldots, A_{k}$ with $\left|A_{i}\right|=a_{i}$. An $\left(a_{1}, \ldots, a_{k}\right)$-ascending permutation is a permutation $\pi$ that ascends within each of the blocks $A_{1}, \ldots, A_{k}$. This is the same as saying that the descent set of $\pi$ is contained in $\left\{a_{1}, a_{1}+a_{2}, \ldots, a_{1}+a_{2}+\right.$ $\left.\cdots+a_{k-1}\right\}$. In this language, the Gessel-Reutenauer bijection is a map from $\left(a_{1}, \ldots, a_{k}\right)$ ascending permutations to ornaments that preserves cycle structure. 
We provide a generalization of the Gessel-Reutenauer bijection to deal with both ascending and descending blocks. Let $A=\left(a_{1}, \ldots, a_{k}\right)$ and $S \subset\{1, \ldots, k\}$. Then an $\left(a_{1}, \ldots, a_{k}, S\right)$-permutation (or just an $(A, S)$-permutation if $a_{1}, \ldots, a_{k}$ are clear from context) is a permutation that descends in the blocks $A_{i}$ for $i \in S$ and ascends in all of the other blocks. We generalize the Gessel-Reutenauer bijection to give a cycle-structurepreserving bijection from the $(A, S)$-permutations to ornaments with certain properties. Our bijection can be thought of as equivalent to Reiner's bijection for signed permutations, as a descent for normal permutations is the same as an ascent over negative values for signed permutations [8].

Both here and in [5], the Gessel-Reutenauer bijection is easy to describe. We take a permutation $\pi$, write it as a product of disjoint cycles, and replace each element of each cycle by the block it belongs to. A permutation and its image under the bijection are illustrated later in the paper, in Figures 2 and 3, respectively. Since the Gessel-Reutenauer bijection forgets so much structure, the surprising thing is that it is injective.

We describe the image of our bijection in Theorem 2.2. In proving Theorem 2.2, we consider a second bijection onto ornaments, but this time the ornaments have properties that are easier to describe. The tradeoff is that the bijection no longer preserves cycle structure, but it is not too difficult to describe how the cycle structure changes. This second bijection is described in Theorem 2.1.

Our bijective methods apply to some of the results in the original paper by Gessel and Reutenauer. The $\left(a_{1}, \ldots, a_{k}\right)$-ascending permutations are all permutations with at most a given descent set. By using inclusion-exclusion on the $\left(a_{1}, \ldots, a_{k}\right)$-ascending permutations, we can study the number of permutations with exactly a given descent set. We can do the same thing with the $\left(a_{1}, \ldots, a_{k}\right)$-descending permutations. It turns out that comparing the two allows us to see what happens when we take the complement of the descent set. In [5], Gessel and Reutenauer prove the following two theorems.

Theorem 4.1 of [5]. Associate to each conjugacy class of $S_{n}$ a partition $\lambda$ based on cycle structure. If $\lambda$ has no parts congruent to 2 modulo 4 and every odd part of $\lambda$ occurs only once, then the number of permutations of cycle structure $\lambda$ with a given descent set is equal to the number of permutations of cycle structure $\lambda$ with the complementary descent set.

(a)

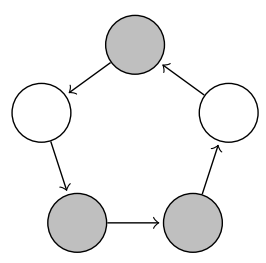

(b)



(c)

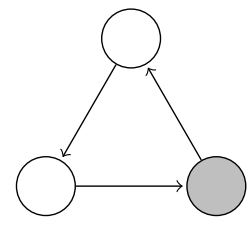

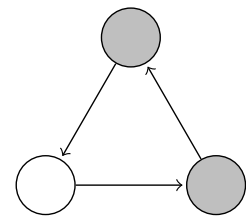

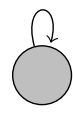

Figure 1: Examples of necklaces and ornaments. (a) and (b) are two different representations of the same necklace with 5 vertices. (c) is an ornament with two different 3-cycles and a 1-cycle. 
Theorem 4.2 of [5]. The number of involutions in $S_{n}$ with a given descent set is equal to the number of involutions in $S_{n}$ with the complementary descent set.

We obtain Theorem 4.1 of [5] as a consequence of Corollary 3.1 by setting $S$ to $\emptyset$. Corollary 3.1 deals with permutations with at least a given ascent or descent set, but as noted before we can apply inclusion-exclusion to get the same result about pemutations with exactly a given ascent or descent set.

Corollary 3.1. Associate to each conjugacy class $\mathcal{C}$ of $S_{n}$ a partition $\lambda$ of $n$ based on cycle structure.

The number of $(A, S)$-permutations in $\mathcal{C}$ is the same if we replace $S$ by $\{1, \ldots, k\} \backslash S$, assuming that all odd parts of $\lambda$ are distinct and $\lambda$ has no parts congruent to 2 mod 4 .

To our knowledge, this is the first bijective proof of Theorem 4.1 of [5]. We also obtain the following generalization of Theorem 4.2 of [5].

Corollary 3.2. The number of $(A, S)$-involutions is the same if we replace $S$ by its complement.

This is the first known bijective proof of Theorem 4.2 of [5].

Our bijections also allow us to take a purely combinatorial approach to the problems considered in [6] and [4]. In [6], Han and Xin, motivated by a problem of Stanley [9], study the $\left(a_{1}, \ldots, a_{k}\right)$-descending derangements, meaning derangements that descend in each of the blocks $A_{1}, \ldots, A_{k}$ (so, in our language, the case when $S=\{1, \ldots, k\}$ ). Han and Xin use symmetric functions to prove their results. In [4], Eriksen, Freij, and Wästlund also study the $\left(a_{1}, \ldots, a_{k}\right)$-descending derangements, but they use generating functions instead of symmetric functions.

Eriksen et al. show that the number of $\left(a_{1}, \ldots, a_{k}\right)$-descending derangements is symmetric in $a_{1}, \ldots, a_{k}$ and ask for a bijective proof of this fact. We obtain a bijective proof of the following stronger statement.

Corollary $4.1^{1}$. Let $\sigma$ be a permutation of $\{1, \ldots, k\}$ and let $\mathcal{C}$ be a conjugacy class in $S_{n}$. The number of $\left(a_{1}, \ldots, a_{k}, S\right)$-permutations in $\mathcal{C}$ is the same as the number of $\left(a_{\sigma(1)}, \ldots, a_{\sigma(k)}, \sigma(S)\right)$-permutations in $\mathcal{C}$.

Eriksen et al. also show that the number of $\left(a_{1}, \ldots, a_{k}\right)$-descending derangements is

$$
\sum_{0 \leqslant b_{m} \leqslant a_{m}, m=1, \ldots, k}(-1)^{\sum b_{i}}\left(\begin{array}{c}
\sum\left(a_{i}-b_{i}\right) \\
a_{1}-b_{1}, \ldots, a_{k}-b_{k}
\end{array}\right) .
$$

They do this using the generating function

$$
\frac{1}{1-x_{1}-\cdots-x_{k}}\left(\frac{1}{1+x_{1}} \cdots \frac{1}{1+x_{k}}\right)
$$

\footnotetext{
${ }^{1}$ Sergei Elizalde proves a slightly less general version of Corollary 4.1 as Proposition 4.2 of [3].
} 
for the $\left(a_{1}, \ldots, a_{k}\right)$-descending derangements, which first appears in [6]. They ask for a combinatorial proof of their formula using inclusion-exclusion. They also ask for a similar enumeration of the $\left(a_{1}, \ldots, a_{k}\right)$-ascending derangements. We provide both of these as a corollary to Theorem 2.1.

Corollary $4.2^{2}$. The number of $(A, S)$-derangements is the coefficient of $x_{1}^{a_{1}} \cdots x_{k}^{a_{k}}$ in

$$
\frac{1}{1-x_{1}-\cdots-x_{k}}\left(\frac{\prod_{i \notin S}\left(1-x_{i}\right)}{\prod_{i \in S}\left(1+x_{i}\right)}\right) \text {. }
$$

Let $l_{m}=a_{m}$ if $m \in S$ and let $l_{m}=1$ otherwise. The number of $(A, S)$-derangements is also

$$
\sum_{0 \leqslant b_{m} \leqslant l_{m}, m=1, \ldots, k}(-1)^{\sum b_{i}}\left(\begin{array}{c}
\sum_{1}\left(a_{i}-b_{i}\right) \\
a_{1}-b_{1}, \ldots, a_{k}-b_{k}
\end{array}\right) .
$$

It is also possible to prove Corollary 4.2 more directly using some structural lemmas about $(A, S)$-derangements and standard techniques in recursive enumeration. We include this approach as well, since it is more in the spirit of the paper by Eriksen, Freij, and Wästlund [4].

We also work towards explaining a polynomial identity in [4]. Let $f_{\lambda}(n)$ be the generating function for permutations on $\{1, \ldots, n\}$ by number of fixed points. In other words, the $\lambda^{k}$ coefficient of $f_{\lambda}(n)$ is the number of permutations in $S_{n}$ with $k$ fixed points. Eriksen et al. prove that the polynomial

$$
\frac{1}{a_{1} ! \cdots a_{k} !} \sum_{T \subset\{1, \ldots, n\}}(-1)^{|T|} f_{\lambda}(|\{1, \ldots, n\} \backslash T|) \prod_{i=1}^{k} f_{\lambda}\left(\left|A_{i} \cap T\right|\right)
$$

is (i) constant and (ii) counts the $\left(a_{1}, \ldots, a_{k}\right)$-descending derangements when $\lambda=1$. Eriksen et al. show that this polynomial is constant by taking a derivative. They then ask for a combinatorial proof that this polynomial always counts the $\left(a_{1}, \ldots, a_{k}\right)$-descending derangements. While we fall short of this goal, we obtain a more combinatorial proof that the polynomial is constant by using a sieve-like argument. We obtain the constant as a sum, which we then generalize to a sum that counts the $(A, S)$-derangements.

The rest of the paper is divided into five sections. In Section 2, we describe the two bijections used in the remainder of the paper and prove that they are bijections. In the process, we introduce maps $\Phi, \Psi$, and $\Upsilon$ that will be useful in later sections. In Section 3 , we provide bijective proofs of Theorems 4.1 and 4.2 from the original Gessel-Reutenauer paper [5].

In Section 4, we provide enumerations of the $(A, S)$-derangements. Section 4 is split into two subsections. In Subsection 4.1, we provide the enumerations using the bijective tools developed in Section 2. In this subsection, we also prove Corollary 4.1. In Subsection

\footnotetext{
${ }^{2}$ The referee points out that this result was presented by Dongsu Kim at Permutation Patterns 2009. Dongsu Kim also presented Theorem 6.2, a result linking the $(A, S)$-derangements to another class of permutations.
} 
4.2, we provide the enumerations again, this time using recursive tools similar to those used in [4].

In Section 5, we show that the polynomial from [4] is constant and derive a new combinatorial sum for the $\left(a_{1}, \ldots, a_{k}\right)$-descending derangements. In Section 6 , we generalize the sum from Section 5 to count the $(A, S)$-derangements. We have tried to make Sections 4.2 through 6 as self-contained as possible, in case the reader is interested only in the case of derangements and not general permutations. Sections 4.2 and 5 are completely self-contained. Section 6 depends on Sections 2 and 5.

In Section 7, we discuss directions of further research, including the further study of the Gessel-Reutenauer map $\Phi$ as well as a generalization of the polynomial identity from [4]. We also define all the terms used in this paper in Section 9, which occurs after the Acknowledgements and before the Bibliography. These terms are all defined either in the introduction or as they appear in the paper, but we have also collected them in a single location for easy reference.

\section{The Two Bijections}

We now describe our two bijections. Here and later, we will have occasion to talk about ornaments labeled by $\{1, \ldots, k\}$. In this case we call the integers 1 through $k$ colors, the elements in $S$ descending colors, and the elements not in $S$ ascending colors.

Also define the fundamental period of a necklace as the smallest contiguous subsequence $P$ of the necklace such that the necklace can be obtained by concatenating $r$ copies of $P$ for some $r$. In this case, the necklace is said to be $r$-repeating. Call an ornament $A$-compatible if its vertices are labeled by $\{1, \ldots, k\}$ and exactly $a_{i}$ vertices are labeled by $i .^{3}$

Our first map is from permutations to $A$-compatible ornaments. It is a map $\Phi$ that takes a permutation, writes it as a product of disjoint cycles, and replaces each element of each cycle by the block it belongs to.

For example, let us suppose that we were considering the $((8,10),\{1\})$-permutationsin other words, permutations that descend in a block of length 8 and then ascend in a block

\footnotetext{
${ }^{3}$ Here and later, we assume for notational convenience that $A=\left(a_{1}, \ldots, a_{k}\right)$, where the $a_{i}$ are all non-negative integers.
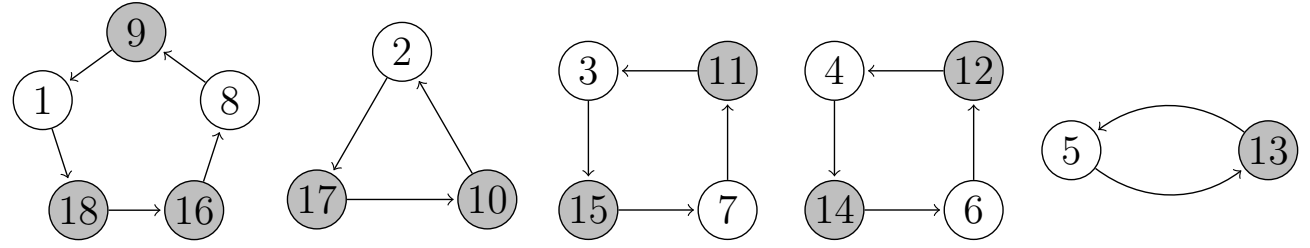

Figure 2: The permutation $\pi=181715141312119123456781016$, written as a product of disjoint cycles. This is the pre-image of the ornament in Figure 3 under the map $\Phi$.
} 
of length 10. In particular, we will take the permutation $\pi=18171514131211912345$ 67810 16. This permutation has cycle structure $\left(\begin{array}{lllll}1 & 18 & 16 & 8 & 9\end{array}\right)\left(\begin{array}{llll}2 & 17 & 10\end{array}\right)\left(\begin{array}{llll}3 & 15 & 7 & 11\end{array}\right)$ (4 14612 )(5 13). We replace each vertex in each cycle by the block it belongs to $\left(A_{1}\right.$ or $\left.A_{2}\right)$ to get $\left(\begin{array}{lllll}1 & 2 & 2 & 1 & 2\end{array}\right)\left(\begin{array}{lll}1 & 2 & 2\end{array}\right)\left(\begin{array}{llll}1 & 2 & 1 & 2\end{array}\right)\left(\begin{array}{llll}1 & 2 & 1 & 2\end{array}\right)\left(\begin{array}{ll}1 & 2\end{array}\right)$, which corresponds to the ornament depicted in Figure 3.

The map $\Phi$ clearly preserves cycle structure. We will show later that $\Phi$ is injective on the $(A, S)$-permutations. In addition to $\Phi$, we will consider two maps $\Psi$ and $\Upsilon$. Before defining $\Psi$, we need the notion of an augmentation of an ornament.

We have illustrated an augmentation of an ornament consisting of a 5-cycle, a 3-cycle, and five 2-cycles in Figure 4 (we will see that it is in fact the image of $\Phi(\pi)$ under $\Psi$ ). Formally, we can think of an ornament $\omega$ as a multiset $\left\{\nu_{1}^{l_{1}}, \ldots, \nu_{m}^{l_{m}}\right\}$, where each $\nu_{i}$ is a cycle and $l_{i}$ is the number of times that $\nu_{i}$ appears in $\omega$. An augmentation of $\omega$ is the multiset $\omega$ together with an $m$-tuple $\lambda=\left(\lambda_{1}, \ldots, \lambda_{m}\right)$, where each $\lambda_{i}$ is a partition of $l_{i}$. We usually denote this augmented ornament by $\omega_{\lambda}$, and we can more concisely represent $\omega_{\lambda}$ by $\left\{\nu_{1}^{\lambda_{1}}, \ldots, \nu_{m}^{\lambda_{m}}\right\}$, since $l_{i}$ is determined by $\lambda_{i}$.

Now we define $\Psi$, which sends ornaments to augmentations of ornaments. The map $\Psi$ takes each cycle $\nu$ in $\omega$ and replaces $\nu$ by $r$ copies of its fundamental period $\rho$, assuming that $\nu$ is $r$-repeating. If there are $n_{r}$ cycles that are $r$-repeating and map to $\rho$, then
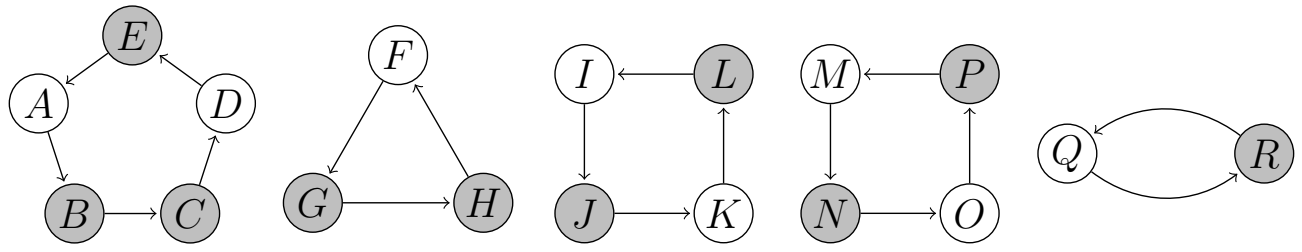

Figure 3: The image of the permutation $\pi=\left(\begin{array}{lllll}1 & 18 & 16 & 8 & 9\end{array}\right)\left(\begin{array}{llll}2 & 17 & 10\end{array}\right)\left(\begin{array}{llll}3 & 15 & 7 & 11\end{array}\right)$ (4 146 12)(5 13) under our bijection. White vertices came from block $A_{1}$ and grey vertices came from block $A_{2}$. The labels $A$ through $R$ are only for the later convenience of referring to specific vertices.
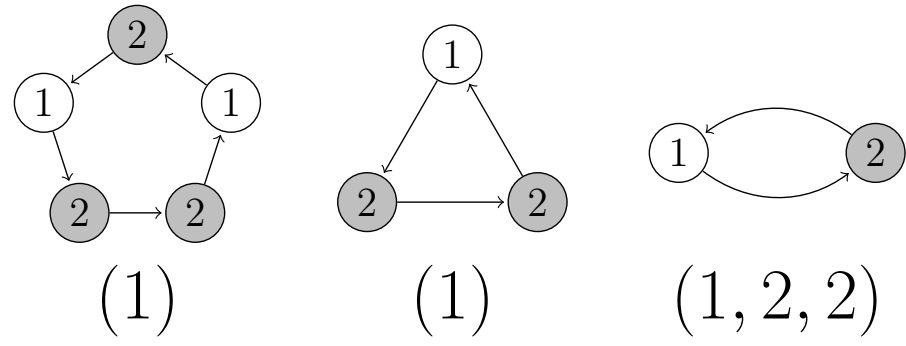

Figure 4: The image of the ornament in Figure 2 under the map $\Psi$. We send the pentagon and triangle each to themselves together with the trivial partition (1). We send the two 4-cycles and the 2-cycle to the 2-cycle together with the partition $(1,2,2)$, since each of these cycles has the same fundamental period and the multiplicities of the periods in the 2-cycle and the two squares are 1, 2, and 2, respectively. 
the partition associated with $\rho$ has $n_{r}$ blocks of size $r$. We also define a map $\Upsilon$ from ornaments to ornaments such that $\Upsilon(\omega)$ is the ornament that $\Psi(\omega)$ augments. We note that all the necklaces in $\Upsilon(\omega)$ are 1-repeating. We will call $A$-compatible ornaments such that all necklaces are 1-repeating A-good ornaments.

Our first result is

Theorem 2.1. The map $\Upsilon \circ \Phi$ is a bijection from $(A, S)$-permutations to A-good ornaments. In particular, every A-good ornament $\omega$ has a unique augmentation $\omega_{\lambda}$ that is in the image of $\Psi \circ \Phi$. If $\omega=\left\{\nu_{1}^{l_{1}}, \ldots, \nu_{m}^{l_{m}}\right\}$, then $\lambda=\left(\lambda_{1}, \ldots, \lambda_{m}\right)$, where

$$
\lambda_{i}= \begin{cases}(1, \ldots, 1), & \text { if } \nu_{i} \text { has an even number of vertices from descending blocks } \\ (2, \ldots, 2), & \text { if } \nu_{i} \text { has an odd number of such vertices and } l_{i} \text { is even } \\ (2, \ldots, 2,1), & \text { if } \nu_{i} \text { has an odd number of such vertices and } l_{i} \text { is odd. }\end{cases}
$$

Theorem 2.1 immediately implies

Theorem 2.2. The map $\Phi$ is an injection from the $(A, S)$-permutations into the $A$ compatible ornaments. The image of $\Phi$ is all A-compatible ornaments satisfying the following three conditions.

1. If the fundamental period of a necklace contains an even number of vertices from descending blocks, then the necklace is 1-repeating.

2. If the fundamental period of a necklace contains an odd number of vertices from descending blocks, then the necklace is either 1-repeating or 2-repeating.

3. If a necklace contains an odd number of vertices from descending blocks, then there are no other necklaces identical to it in the ornament.

Our main tool in proving Theorem 2.1 will be two sequences that we associate with a vertex of an ornament.

Given a vertex $v$, define the sequence $W(v)=\left\{w_{0}(v), w_{1}(v), \ldots\right\}$ by $w_{0}(v)=v$, $w_{i+1}(v)=s\left(w_{i}(v)\right)$, where $s(x)$ is the successor of $x$ in the necklace. Thus $w_{0}, w_{1}, \ldots$ is the sequence of colors one encounters if one starts at the vertex $v$ and walks along the necklace containing $v$.

Similarly define the sequence $A(v)=\left\{a_{0}(v), a_{1}(v), \ldots\right\}$ by $a_{i}(v)=(-1)^{r_{i}(v)} w_{i}(v)$, where $r_{i}(v)$ is the number of vertices in $\left\{w_{0}(v), \ldots, w_{i-1}(v)\right\}$ that come from descending blocks.

We call $W(v)$ the walk from $v$ and $A(v)$ the signed walk from $v$. Table 1 gives the sequences $A(v)$ for $v=A, \ldots, R$ for the ornament in figure 3 . For convenience, we prove the following:

Lemma 2.3. Let $v$ and $v^{\prime}$ be two vertices. Their walks $W(v)$ and $W\left(v^{\prime}\right)$ agree up through $w_{i}$ if and only if their signed walks $A(v)$ and $A\left(v^{\prime}\right)$ agree up through $a_{i}$. 
Proof. If their signed walks agree up through $a_{i}$, their walks must agree up through $w_{i}$, since $a_{i}= \pm w_{i}$ and $w_{i}>0$ always.

Now suppose their walks agree up through $w_{i}$. Then $r_{j}(v)=r_{j}\left(v^{\prime}\right)$ for all $j \leqslant i$ and $w_{j}(v)=w_{j}\left(v^{\prime}\right)$ for all $j \leqslant i$, so $(-1)^{r_{j}(v)} w_{j}(v)=(-1)^{r_{j}\left(v^{\prime}\right)} w_{j}\left(v^{\prime}\right)$ for all $j \leqslant i$. This is the same as saying that $a_{j}(v)=a_{j}\left(v^{\prime}\right)$ for all $j \leqslant i$, so we are done.

The key observation about $W(v)$ and $A(v)$ is given in the following lemma and its corollary.

Lemma 2.4. If two vertices $v$ and $v^{\prime}$ have sequences of colors that agree through $w_{l-1}$, then the order of $v$ and $v^{\prime}$ is determined by the order of $w_{l}(v)$ and $w_{l}\left(v^{\prime}\right)$. In fact, if $\left\{w_{1}, \ldots, w_{l-1}\right\}$ has an even number of vertices from descending blocks, then $v$ and $v^{\prime}$ come in the same order as $w_{l}(v)$ and $w_{l}\left(v^{\prime}\right)$. Otherwise, they come in the opposite order.

Corollary 2.5. The vertices $v$ and $w$ come in the same order as $A(v)$ and $A(w)$, if we consider the latter pair in the lexicographic order.

Proof of Lemma 2.4. We need to show that if the walks from $v$ and $v^{\prime}$ agree through $w_{l-1}$, then $v$ and $v^{\prime}$ come in the same order as $(-1)^{r_{l}(v)} w_{l}(v)$ and $(-1)^{r_{l}\left(v^{\prime}\right)} w_{l}\left(v^{\prime}\right)$.

We proceed by induction on $l$. In the base case $l=1$, the result is a consequence of the fact that $v$ and $v^{\prime}$ come from the same block, and if that block is ascending then $v$ and $v^{\prime}$ are in the same order as their successors, whereas if it is descending they are in the opposite order.

Now suppose that $v$ and $v^{\prime}$ have sequences of colors that agree through $w_{l}$. Then they also agree through $w_{l-1}$, so by the inductive hypothesis $v$ and $v^{\prime}$ come in the same order as $(-1)^{r_{l}(v)} w_{l}(v)$ and $(-1)^{r_{l}\left(v^{\prime}\right)} w_{l}\left(v^{\prime}\right)$ since $w_{l}(v)$ and $w_{l}\left(v^{\prime}\right)$ have the same color. By taking the case $l=1$ applied to $w_{l}(v)$ and $w_{l}\left(v^{\prime}\right)$, we know that $w_{l}(v)$ and $w_{l}\left(v^{\prime}\right)$ come in the same order as $(-1)^{r_{1}\left(w_{l}(v)\right)} w_{l+1}(v)$ and $(-1)^{r_{1}\left(w_{l}\left(v^{\prime}\right)\right)} w_{l+1}\left(v^{\prime}\right)$. Hence $v$ and $v^{\prime}$ come in the same order as $(-1)^{r_{l}(v)+r_{1}\left(w_{l}(v)\right)} w_{l+1}(v)$ and $(-1)^{r_{l}\left(v^{\prime}\right)+r_{1}\left(w_{l}\left(v^{\prime}\right)\right)} w_{l+1}\left(v^{\prime}\right)$. Since $r_{l}(v)+r_{1}\left(w_{l}(v)\right)=r_{l+1}(v)$, the lemma follows.

Proof of Corollary 2.5. Suppose that $A(v)<A\left(v^{\prime}\right)$ lexicographically. Then there exists an $l$ such that $A(v)$ and $A\left(v^{\prime}\right)$ first differ in the $l$ th position, so the signed walks from $v$ and $v^{\prime}$ agree through $a_{l-1}$. By Lemma 2.3, this means that the walks from $v$ and $v^{\prime}$ agree through $w_{l-1}$, so $v$ and $v^{\prime}$ come in the same order as $a_{l}(v)$ and $a_{l}\left(v^{\prime}\right)$. But $a_{l}(v)<a_{l}\left(v^{\prime}\right)$ by assumption, so $v<v^{\prime}$, as was to be shown.

We are now ready to prove Theorem 2.1.

Proof of Theorem 2.1. We first show that $\Upsilon \circ \Phi$ is a bijection from $(A, S)$-permutations to $A$-compatible ornaments.

To get from an $A$-good ornament $\omega_{0}$ to an ornament $\omega$ in $\Upsilon^{-1}\left(\omega_{0}\right)$, we can do the following. For each set of identical necklaces $\nu^{l}$ in the ornament $\omega_{0}$, split $\nu^{l}$ into $|\nu|$ sets that we will call packets. Each packet consists of the $l$ elements from identical positions in the $l$ necklaces (this notion is well-defined since each necklace in $\omega_{0}$ is 1-repeating). Within each packet, re-choose the successors of each vertex (by permuting them arbitrarily). It is 
easy to verify that this operation preserves the fundamental period of a necklace, so that we end up with an element in $\Upsilon^{-1}\left(\omega_{0}\right)$. It is also easy to see that we can get any element of $\Upsilon^{-1}\left(\omega_{0}\right)$ in this way.

To get from $\omega$ to an element of $\Phi^{-1}(\omega)$, take $\omega$ and then replace, for each $i$, the vertices colored $i$ by the elements of $A_{i}$.

We can then think of $\Phi^{-1} \circ \Upsilon^{-1}$ as follows. Take an $A$-good ornament $\omega_{0}$, and again split $\nu^{l}$ into packets. Now label the vertices of $\omega_{0}$ by the integers 1 through $n$ such that the vertices colored $i$ are labeled by the elements of $A_{i}$. Finally, choose the successors of each vertex. This process is illustrated in Figures 5, 6, and 7.

Note that we can recover each of the walks (and hence signed walks) of $\omega$ using just $\Upsilon(\omega)$. By Corollary 2.5, then, there is only one labeling of the vertices of $\omega_{0}$ that can yield an $(A, S)$-permutation. It is obtained by first listing the vertices $v_{1}, \ldots, v_{n}$ of the template so that if $i<j$ then $A\left(v_{i}\right)<A\left(v_{j}\right)$; then labeling $v_{i}$ with the integer $i$ (ties in $A(v)$ are irrelevant here, since the later re-assignment of successors makes all vertices with the same walk symmetric with respect to each other).

Once we have done this, there is a unique way to pick the successors of each vertex to get an $(A, S)$-permutation. If a packet comes from an ascending block, then the successors of the vertices should be ordered in the same way as the vertices themselves. If a packet comes from a descending block, then the successors of the vertices should be ordered in the opposite way as the vertices themselves. This constraint uniquely determines the successors of each vertex, and we can also see that this constraint is sufficient to get an $(A, S)$-permutation. We have thus shown that, for any $A$-good ornament $\omega_{0}$, there is a unique element $\pi$ of $(\Upsilon \circ \Phi)^{-1}$ that is also an $(A, S)$-permutation.

We now consider the cycle structure of this $(A, S)$-permutation (this will let us determine the image of $\Psi \circ \Phi)$.

Suppose $\omega_{0}$ has a set of necklaces $\nu^{l}$, and $\nu$ has $d$ vertices from descending blocks and $x$ vertices in total. If $d$ is even, then $\nu^{l}$ will contribute $l$ cycles, all of length $x$, to $\pi$. If $d$ is odd, then we will instead end up with cycles of length $2 x$. The exception is if $l$ is odd, in which case there is also one cycle of length $x$ coming from the vertices in each packet that take on the median value for that packet.

This cycle structure corresponds precisely to the augmentation described in the statement of Theorem 2.1, so we are done.

Table 1: The first 7 terms of $A(v)$ for $v=A, \ldots, R$. We have ordered the entries lexicographically by $A(v)$.

\begin{tabular}{|c|c|c|c|c|c|}
\hline $\mathrm{A}$ & $1,-2,-2,-1, \quad 2, \quad 1,-2$ & $\mathrm{Q}$ & $1,-2,-1, \quad 2, \quad 1,-2,-1$ & $\mathrm{~N}$ & $2, \quad 1,-2,-1, \quad 2, \quad 1,-2$ \\
\hline $\mathrm{F}$ & $1,-2,-2,-1, \quad 2, \quad 2, \quad 1$ & $\mathrm{D}$ & $1,-2,-1, \quad 2, \quad 2, \quad 1,-2$ & $\mathrm{P}$ & $2, \quad 1,-2,-1, \quad 2, \quad 1,-2$ \\
\hline I & $1,-2,-1, \quad 2$, & $\mathrm{E}$ & $2, \quad 1,-2,-2,-1, \quad 2$ & $\mathrm{R}$ & $2, \quad 1,-2,-1, \quad 2, \quad 1,-2$ \\
\hline $\mathrm{K}$ & $1,-2,-1, \quad 2$ & $\mathrm{H}$ & $2, \quad 1,-2,-2,-1, \quad 2, \quad 2$ & $\mathrm{C}$ & $2, \quad 1,-2,-1, \quad 2, \quad 2, \quad 1$ \\
\hline $\mathrm{M}$ & $1,-2,-1, \quad 2$ & $\mathrm{~J}$ & $2, \quad 1,-2,-1, \quad 2, \quad 1,-2$ & G & $2,2,1,-2,-2,-1$, \\
\hline & $1,-2,-1, \quad 2, \quad 1,-2,-1$ & $\mathrm{~L}$ & $2, \quad 1,-2,-1$ & B & $1,-2,-1, \quad 2, \quad 2$ \\
\hline
\end{tabular}




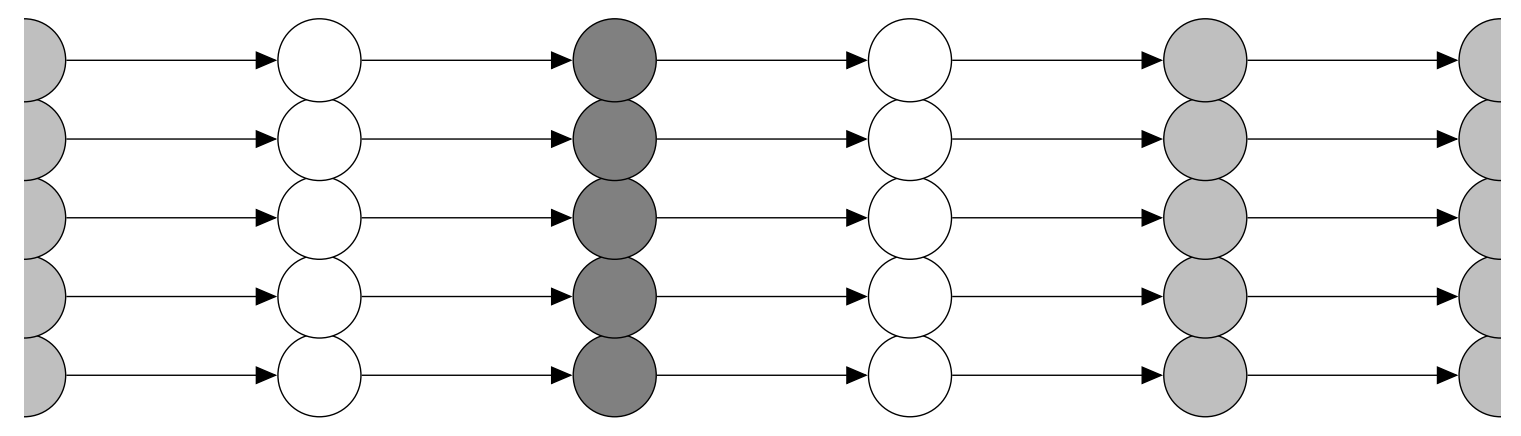

Figure 5: An ornament $\omega_{0}$ with 5 necklaces, each with 5 vertices. Each column is a packet of $\omega_{0}$. The first and last half-vertex are the same. Light grey indicates block 1, white indicates block 2 , and dark grey indicates block 3 , so $A=(10,10,5)$. Also, $S=\{1,3\}$, so blocks 1 and 3 descend while block 2 ascends. The arrows indicate successors in $\omega_{0}$.

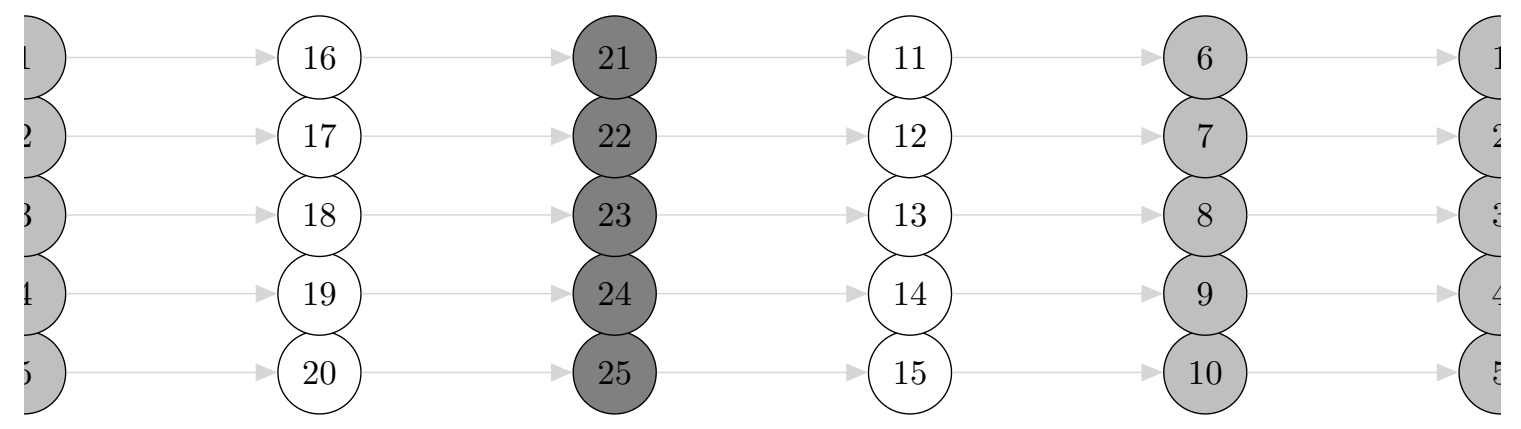

Figure 6: The unique way of numbering the vertices in the ornament from Figure 5 to get an $(A, S)$-permutation, based on Corollary 2.5.

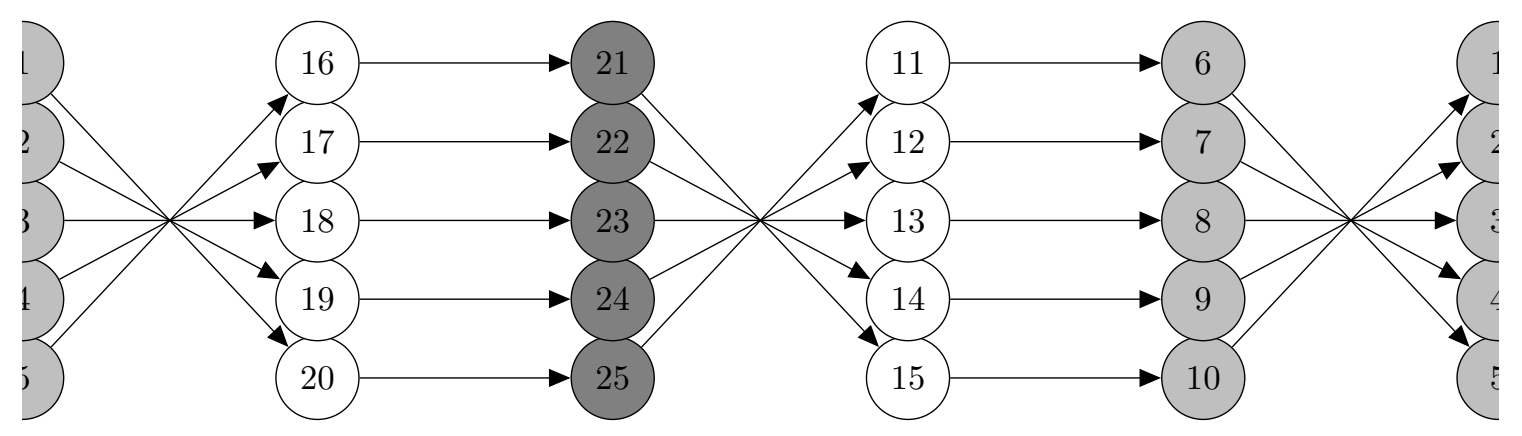

Figure 7: The unique way of choosing successors for the numbered ornament in Figure 6 to yield an $(A, S)$-permutation. The successors are indicated by arrows. Observe that we end up with the 5-cycle ( $\left.\begin{array}{lllll}3 & 18 & 23 & 13 & 8\end{array}\right)$ and two 10-cycles. 
In future sections we will deal with the $(A, S)$-derangements. For this, the following proposition will be helpful.

Proposition 2.6. The image of the $(A, S)$-derangements under $\Upsilon \circ \Phi$ is all $A$-good ornaments with no 1-cycles from ascending blocks and an even number of 1-cycles from each descending block.

Proof. This follows immediately by considering the formula for $\lambda_{i}$ at the end of the statement of Theorem 2.1. An augmentation of an ornament corresponds to a derangement if and only if no necklaces of size 1 are augmented by partitions with parts of size 1 .

We record the following corollary for use in Section 6 .

Corollary 2.7. The $(A, S)$-derangements are in bijection with A-compatible ornaments satisfying the following properties:

- Every cycle is either 1-repeating or 2-repeating.

- The only 2-repeating cycles are monochromatic 2-cycles from a descending block.

- There are no 1-cycles.

Proof. We can apply Proposition 2.6, then replace every pair of 1-cycles from a descending block with a 2-cycle from the same block.

\section{Revisiting Gessel and Reutenauer}

In this section, we present two corollaries of the results presented in Section 2. They imply Theorems 4.1 and 4.2 of [5].

Corollary 3.1. Associate to each conjugacy class $\mathcal{C}$ of $S_{n}$ a partition $\lambda$ of $n$ based on cycle structure.

The number of $(A, S)$-permutations in $\mathcal{C}$ is the same if we replace $S$ by $\{1, \ldots, k\} \backslash S$, assuming that all odd parts of $\lambda$ are distinct and $\lambda$ has no parts congruent to 2 mod 4 .

Proof. We will take an ornament that satisfies the conditions of Theorem 2.2, then show that it still satisfies the conditions of Theorem 2.2 if we make each ascending block a descending block and vice versa. This would provide an injection from the $(A, S)$ permutations in $\mathcal{C}$ and the $(A,\{1, \ldots, k\} \backslash S)$-permutations in $\mathcal{C}$. Since taking the complement of $S$ twice yields $S$ again, this is sufficient.

Suppose we have an ornament $\omega$ that satisfies the conditions of Theorem 2.2. Then (i) every necklace with an even number of vertices from descending blocks in its fundamental period is 1-repeating, (ii) every necklace with an odd number of vertices from descending blocks in its fundamental period is either 1-repeating or 2-repeating, and (iii) no two necklaces with an odd number of vertices from descending blocks are isomorphic. 
If a necklace has an even number of total vertices, then the conditions on $\lambda$ ensure that the number of vertices in the cycle is divisible by 4 . Since every necklace is at most 2-repeating, this means that the size of the fundamental period must be even. In this case, the number of vertices from ascending and descending blocks in the fundamental period has the same parity. Therefore, whether the necklace satisfies the hypotheses of (i), (ii), and (iii) remains unchanged when we replace $S$ by its complement; since we are left with the same necklace, whether that necklace satisfies the conclusions of (i), (ii), and (iii) also remains unchanged.

If a necklace has an odd number of total vertices, then the conditions on $\lambda$ imply that it is the only necklace with that many vertices and thus cannot be isomorphic to any other necklace. Thus the conclusion of (iii) is automatically satisfied. The necklace also cannot be 2-repeating, since it has an odd number of total vertices, so the conclusions of (i) and (ii) combine to say that, in all cases, the necklace must be 1-repeating. This condition is independent of $S$, so whether this necklace satisfies the conditions imposed by (i), (ii), and (iii) does not change if we replace $S$ by its complement.

We have shown that an ornament satisfying the conditions of Theorem 2.2 will still do so if we replace $S$ by its complement, so we are done.

Corollary 3.2. The number of $(A, S)$-involutions is the same if we replace $S$ by its complement.

Proof. Under the map $\Upsilon \circ \Phi$, the $(A, S)$-involutions are in bijection with $A$-compatible ornaments such that (i) there are only 1-cycles and 2-cycles; (ii) any 2-cycle has vertices of distinct colors; and (iii) if a 2-cycle has exactly one vertex from a descending block, then it is not isomorphic to any other 2-cycle.

We observe that if we replace $S$ by its complement, then condition (ii) does not change, since any cycle with exactly one descending vertex also has exactly one ascending vertex. Also, conditions (i) and (iii) do not change because they have nothing to do with whether a block is ascending or descending. Therefore, the ornaments that correspond to $(A, S)$-involutions also correspond to $(A,\{1, \ldots, k\} \backslash S)$-involutions. We can replace $S$ with $\{1, \ldots, k\} \backslash S$ in the preceeding argument to see that it is also the case that the ornaments corresponding to $(A,\{1, \ldots, k\} \backslash S)$-involutions also correspond to $(A, S)$-permutations, so we are done.

\section{Enumerating the $(A, S)$-derangements}

\subsection{Bijective enumeration of the $(A, S)$-derangements}

In this subsection, we use the results from Section 2 to enumerate the $(A, S)$-derangements.

Corollary 4.1. Let $\sigma$ be a permutation of $\{1, \ldots, k\}$ and let $\mathcal{C}$ be a conjugacy class in $S_{n}$. The number of $\left(a_{1}, \ldots, a_{k}, S\right)$-permutations in $\mathcal{C}$ is the same as the number of $\left(a_{\sigma(1)}, \ldots, a_{\sigma(k)}, \sigma(S)\right)$-permutations in $\mathcal{C}$. 
Proof. The description of the image of $\Phi$ in Theorem 2.2 doesn't distinguish between the blocks.

Corollary 4.2. The number of $(A, S)$-derangements is the coefficient of $x_{1}^{a_{1}} \cdots x_{k}^{a_{k}}$ in

$$
\frac{1}{1-x_{1}-\cdots-x_{k}}\left(\frac{\prod_{i \notin S}\left(1-x_{i}\right)}{\prod_{i \in S}\left(1+x_{i}\right)}\right) \text {. }
$$

Let $l_{m}=a_{m}$ if $m \in S$ and let $l_{m}=1$ otherwise. The number of $(A, S)$-derangements is also

$$
\sum_{0 \leqslant b_{m} \leqslant l_{m}, m=1, \ldots, k}(-1)^{\sum b_{i}}\left(\begin{array}{c}
\sum\left(a_{i}-b_{i}\right) \\
a_{1}-b_{1}, \ldots, a_{k}-b_{k}
\end{array}\right) .
$$

Proof. As in Section 2, we will refer to an $A$-compatible ornament where every necklace is 1-repeating as an $A$-good ornament.

First note that

$$
\frac{1}{1-x_{1}-\cdots-x_{k}}=\sum_{n=0}^{\infty}\left(\sum_{i=1}^{k} x_{i}\right)^{n}=\sum_{c_{1}, \ldots, c_{k}=0}^{\infty}\left(\begin{array}{c}
c_{1}+\cdots+c_{k} \\
c_{1}, \ldots, c_{k}
\end{array}\right) x_{1}^{c_{1}} \cdots x_{k}^{c_{k}} .
$$

From here it is easy to see that the $x_{1}^{a_{1}} \cdots x_{k}^{a_{k}}$ coefficient in (1) is equal to the sum given in (2). It thus suffices to establish that (2) enumerates the $(A, S)$-derangements.

By Proposition 2.6, the $(A, S)$-derangements are in bijection with the $A$-good ornaments with no 1-cycles in ascending colors and an even number of 1-cycles in each descending color.

Note that the number of $\left(a_{1}, \ldots, a_{k}\right)$-good ornaments is $\left(\begin{array}{c}a_{1}+\cdots+a_{k} \\ a_{1}, \ldots, a_{k}\end{array}\right)$. This is because these ornaments are in bijection with the $\left(a_{1}, \ldots, a_{k}\right)$-ascending permutations by Theorem 2.2. There are $\left(\begin{array}{c}a_{1}+\cdots+a_{k} \\ a_{1}, \ldots, a_{k}\end{array}\right)\left(a_{1}, \ldots, a_{k}\right)$-ascending permutations because, once we determine the set of permutation values within each block, there is exactly one way to order them to be increasing.

Also, the number of $\left(a_{1}, \ldots, a_{k}\right)$-good ornaments with at least $b_{i} 1$-cycles of color $i$ is $\left(\begin{array}{c}\left(a_{1}-b_{1}\right)+\cdots+\left(a_{k}-b_{k}\right) \\ a_{1}-b_{1}, \ldots, a_{k}-b_{k}\end{array}\right)$. This is because they are in bijection with the $\left(a_{1}-b_{1}, \ldots, a_{k}-b_{k}\right)$-good ornaments (the bijection comes from removing, for each $i, b_{i}$ of the 1-cycles of color $i$ ).

Now if $f\left(b_{1}, \ldots, b_{k}\right)$ is the number of $\left(a_{1}, \ldots, a_{k}\right)$-good ornaments with at least $b_{i} 1$ cycles of color $i$, then a standard inclusion-exclusion argument shows that the number of ornaments with an even number of 1-cycles in descending colors and no 1-cycles in ascending colors is

$$
\sum_{0 \leqslant b_{m} \leqslant l_{m}, m=1, \ldots, k}(-1)^{\sum b_{i}} f\left(b_{1}, \ldots, b_{k}\right)
$$

where $l_{m}=a_{m}$ if $m \in S$ and $l_{m}=1$ if $m \notin S$. Since we know that $f\left(b_{1}, \ldots, b_{k}\right)=$ $\left(\begin{array}{c}\left(a_{1}-b_{1}\right)+\cdots+\left(a_{k}-b_{k}\right) \\ a_{1}-b_{1}, \ldots, a_{k}-b_{k}\end{array}\right),(2)$ follows. 


\subsection{Recursive enumeration of the $(A, S)$-derangements}

In this subsection, we will enumerate the $(A, S)$-derangements using recursive techniques. We will refer to an index $i, 1 \leqslant i \leqslant n$, such that $\pi(i)<i$ as a deficiency, and an index with $\pi(i)>i$ as an excedance. We let $\operatorname{Des}(\pi)$ denote the descent set of $\pi, \operatorname{Exc}(\pi)$ the set of excedances, and $\operatorname{Fix}(\pi)$ the set of fixed points.

We begin by describing a process of "fixed point removal" defined in Sections 1 and 2 of [4]. This process preserves descents, excedances, and fixed points (and so also ascents and deficiencies).

Lemma 4.3. Given integers $i$ and $j, j \neq i$, define

$$
\rho_{i}(j)= \begin{cases}j & \text { if } j<i \\ j-1 & \text { if } j>i\end{cases}
$$

Given a set $S$ of integers, define $\rho_{i}(S)$ to be $\rho_{i}(S \backslash\{i\})$. For a permutation $\pi$ on $\{1, \ldots, n\}$ with $\pi(i)=i$, define the permutation $\psi_{i}(\pi)$ on $\{1, \ldots, n-1\}$ as $\psi_{i}(\pi)=\rho_{i} \pi \rho_{i}^{-1}$.

The map $\psi_{i}$ is a bijection from permutations on $\{1, \ldots, n\}$ with $\pi(i)=i$ to permutations on $\{1, \ldots, n-1\}$. Furthermore, $\operatorname{Des}\left(\psi_{i}(\pi)\right)=\rho_{i}(\operatorname{Des}(\pi)), \operatorname{Exc}\left(\psi_{i}(\pi)\right)=\rho_{i}(\operatorname{Exc}(\pi))$, and $\operatorname{Fix}\left(\psi_{i}(\pi)\right)=\rho_{i}(\operatorname{Fix}(\pi))$.

The proof is a routine verification, so we omit it. The easiest way to visualize this process is to think of permutations in terms of their permutation matrices, and then $\psi_{i}(\pi)$ is the permutation we get if we remove the $i$ th row and $i$ th column of $\pi$. We refer to the process of sending $\pi$ to $\psi_{i}(\pi)$ as "removing the fixed point $i$ from $\pi$."

The next lemma appears implicitly in both [6] and [4].

Lemma 4.4. If $i \in S$, then any $\left(a_{1}, \ldots, a_{k}, S\right)$-permutation has at most one fixed point in the block $A_{i}$.

Proof. The permutation values are decreasing in $A_{i}$, so if $j \in A_{i}$ and $\pi(j)=j$, then all elements of $A_{i}$ coming before $j$ are excedances, and all elements of $A_{i}$ coming after $j$ are deficiencies.

This implies the following bijection, which appears as Lemma 2.2 of [4]. We include the proof for completeness.

Lemma 4.5. If $i \in S$, then there is a bijection between $\left(a_{1}, \ldots, a_{i}, \ldots, a_{k}, S\right)$-permutations with one fixed point in $A_{i}$ and $\left(a_{1}, \ldots, a_{i}-1, \ldots, a_{k}, S\right)$-permutations with no fixed points in $A_{i}$.

Proof. To get from a permutation with one fixed point in $A_{i}$ to one with no fixed points in $A_{i}$, just remove the fixed point as explained in Lemma 4.3.

To go backwards, find the unique index $j \in A_{i}$ such that $\pi(j)<j$ but $\pi(k)>k$ for all $k \in A_{i}$ with $k<j$. Then insert a fixed point just before $j$ (by applying $\psi_{j}^{-1}$ to the permutation). In the case that $\pi(k)>k$ for all $k \in A_{i}$, insert a fixed point just after the end of the block $A_{i}$. 
We will also need versions of Lemmas 4.4 and 4.5 to deal with the case of ascending blocks (when $i \notin S$ ).

Lemma 4.6. Let $i \notin S$, and let $\pi$ be an $\left(a_{1}, \ldots, a_{k}, S\right)$-permutation. Then all the fixed points in $A_{i}$ appear consecutively.

Proof. If $j$ is an excedance, $j<k$, and $j, k \in A_{i}$, then $k$ is also an excedance. Similarly, if $j$ is a deficiency, $k<j$, and $j, k \in A_{i}$, then $k$ is also a deficiency.

Lemma 4.7. If $i \notin S$, then there is a bijection between $\left(a_{1}, \ldots, a_{i}, \ldots, a_{k}, S\right)$-permutations with exactly $p$ fixed points in $A_{i}$ and $\left(a_{1}, \ldots, a_{i}-l, \ldots, a_{k}, S\right)$-permutations with exactly $p-l$ fixed points in $A_{i}$. In particular, there is a bijection between $\left(a_{1}, \ldots, a_{i}, \ldots, a_{k}, S\right)$ permutations with exactly $l$ fixed points in $A_{i}$ and $\left(a_{1}, \ldots, a_{i}-l, a_{k}, S\right)$-permutations with exactly zero fixed points in $A_{i}$.

Proof. To get from a permutation with $p$ fixed points in $A_{i}$ to a permutation with $p-l$ fixed points in $A_{i}$, just remove the first $l$ fixed points.

To go backwards, find the unique index $j \in A_{i}$ such that $\pi(j) \geqslant j$ but $\pi(k)<k$ for all $k \in A_{i}$ with $k<j$. Then insert $l$ fixed points just before $j$ (by applying $\psi_{j}^{-1}$ to the permutation $l$ times). In the case that $\pi(k)<k$ for all $k \in A_{i}$, insert $l$ fixed points at the end of the block $A_{i}$.

Note that Lemma 4.7 also holds if we replace all instances of "exactly" with "at least."

Lemmas 4.5 and 4.7 allow us to construct a recursion for the number of $\left(a_{1}, \ldots, a_{k}, S\right)$ derangements. In fact, now that we have Lemma 4.7 in hand, the recursion follows by the same methods as in [4]. For notational convenience, we will assume $S$ to be fixed throughout the argument. Then let $f_{j}\left(a_{1}, \ldots, a_{k}\right)$ denote the number of $\left(a_{1}, \ldots, a_{k}, S\right)$ permutations with no fixed points in blocks $A_{i}$ for $i \leqslant j$. In this case, $f_{k}\left(a_{1}, \ldots, a_{k}\right)$ is the number of $\left(a_{1}, \ldots, a_{k}, S\right)$-derangements.

Proposition 4.8. Let $m_{i}=1$ if $i \in S$ and let $m_{i}=c_{i}$ if $i \notin S$. Then, for all $0 \leqslant j<k$,

$$
f_{j}\left(c_{1}, \ldots, c_{k}\right)=\sum_{h=0}^{m_{j+1}} f_{j+1}\left(c_{1}, \ldots, c_{j}, c_{j+1}-h, c_{j+2}, \ldots, c_{k}\right) .
$$

Proof. The number of $\left(c_{1}, \ldots, c_{k}, S\right)$-permutations with no fixed points in blocks $A_{i}$ for $i \leqslant j$ is the sum, over all $h$, of the number of $\left(c_{1}, \ldots, c_{k}, S\right)$-permutations with no fixed points in blocks $A_{i}$ for $i \leqslant j$ and $h$ fixed points in $A_{j+1}$.

If $j+1 \in S$, then the number of $\left(c_{1}, \ldots, c_{k}, S\right)$-permutations with no fixed points in blocks $A_{i}$ for $i \leqslant j$ and $h$ fixed points in $A_{j+1}$ is equal to 0 if $h>1$. If $h \leqslant 1$, then by Lemma 4.5 the number of such permutations is equal to the number of $\left(c_{1}, \ldots, c_{j+1}-\right.$ $\left.h, \ldots, c_{k}, S\right)$-permutations with no fixed points in blocks $A_{i}$ for $i \leqslant j+1$. But the latter quantity is just $f_{j+1}\left(c_{1}, \ldots, c_{j+1}-h, \ldots, c_{k}\right)$, so in the case that $j+1 \in S$ we have

$$
f_{j}\left(c_{1}, \ldots, c_{k}\right)=\sum_{h=0}^{1} f_{j+1}\left(c_{1}, \ldots, c_{j+1}-h, \ldots, c_{k}\right),
$$


which agrees with Proposition 4.8 .

If $j+1 \notin S$, then the number of $\left(c_{1}, \ldots, c_{k}, S\right)$-permutations with no fixed points in blocks $A_{i}$ for $i \leqslant j$ and $h$ fixed points in $A_{j+1}$ is equal, by Lemma 4.7, to the number of $\left(c_{1}, \ldots, c_{j+1}-h, \ldots, c_{k}, S\right)$-permutations with no fixed points in blocks $A_{i}$ for $i \leqslant j+1$. This latter quantity is again just $f_{j+1}\left(c_{1}, \ldots, c_{j+1}-h, \ldots, c_{k}\right)$, so in the case that $j+1 \notin S$ we have

$$
f_{j}\left(c_{1}, \ldots, c_{k}\right)=\sum_{h=0}^{c_{j+1}} f_{j+1}\left(c_{1}, \ldots, c_{j+1}-h, \ldots, c_{k}\right),
$$

which again agrees with Proposition 4.8. We have thus established Proposition 4.8 in both the ascending and descending cases, so we are done.

We now use Proposition 4.8 to obtain another proof of Corollary 4.2.

Proof of Corollary 4.2. We have already shown that the generating function for the number of $(A, S)$-derangements implies the summation. It therefore suffices to establish the generating function for the $(A, S)$-derangements.

Let

$$
F_{j}\left(x_{1}, \ldots, x_{k}\right)=\sum_{a_{1}, \ldots, a_{k}=0}^{\infty} f_{j}\left(a_{1}, \ldots, a_{k}\right) x_{1}^{a_{1}} \cdots x_{k}^{a_{k}}
$$

be the generating function for $f_{j}\left(a_{1}, \ldots, a_{k}\right)$. We will prove inductively that

$$
F_{j}\left(x_{1}, \ldots, x_{k}\right)=\frac{1}{1-x_{1}-\cdots-x_{k}}\left(\frac{\prod_{i \notin S, i \leqslant j} 1-x_{i}}{\prod_{i \in S, i \leqslant j} 1+x_{i}}\right) .
$$

From this, we will have

$$
F_{k}\left(x_{1}, \ldots, x_{k}\right)=\frac{1}{1-x_{1}-\cdots-x_{k}}\left(\frac{\prod_{i \notin S} 1-x_{i}}{\prod_{i \in S} 1+x_{i}}\right),
$$

which is what we are trying to show.

We start by establishing (3) in the case that $j=0$. When $j=0, f_{j}\left(a_{1}, \ldots, a_{k}\right)$ is just the number of $\left(a_{1}, \ldots, a_{k}, S\right)$-permutations (with no restrictions on fixed points). Thus $f_{0}\left(a_{1}, \ldots, a_{k}\right)=\left(\begin{array}{c}a_{1}+\cdots+a_{k} \\ a_{1}, \ldots, a_{k}\end{array}\right)$, since once we have distributed the numbers $1, \ldots, n$ among the blocks $A_{1}, \ldots, A_{k}$, there is a unique way to order them so that they ascend or descend as they are supposed to. So when $j=0$ we have

$$
\begin{aligned}
F_{0}\left(x_{1}, \ldots, x_{k}\right) & =\sum_{a_{1}, \ldots, a_{k}=0}^{\infty}\left(\begin{array}{c}
a_{1}+\cdots+a_{k} \\
a_{1}, \ldots, a_{k}
\end{array}\right) x_{1}^{a_{1}} \cdots x_{k}^{a_{k}} \\
& =\sum_{n=0}^{\infty}\left(x_{1}+\cdots+x_{k}\right)^{n} \\
& =\frac{1}{1-x_{1}-\cdots-x_{k}} .
\end{aligned}
$$


This completes the base case for the induction. Now the recursive formula for $f_{j}$ in Proposition 4.8 implies that $F_{j}=\frac{F_{j+1}}{1-x_{j+1}}$ if $j+1 \in S$ and $F_{j}=\left(1+x_{j+1}\right) F_{j+1}$ if $j+1 \notin S$, which proves the inductive step. We are therefore done.

Remark. When $S=\emptyset$ (that is, in the case of $\left(a_{1}, \ldots, a_{k}\right)$-ascending permutations), we can also derive the sum in Corollary 4.2 combinatorially. By Lemma 4.7, we can interpret the multinomial coefficient $\left(\begin{array}{c}\sum_{i=1}^{k}\left(a_{i}-b_{i}\right) \\ a_{1}-b_{1}, \ldots, a_{k}-b_{k}\end{array}\right)$ as the number of $(A, \emptyset)$-permutations with at least $b_{i}$ fixed points in block $i$. Then the sum in Corollary 4.2 is an inclusion-exclusion sum that counts the number of $(A, \emptyset)$-permutations with no fixed points in any block, which is the definition of an $\left(a_{1}, \ldots, a_{k}\right)$-ascending derangement.

\section{A polynomial sum}

In this section we study a polynomial sum appearing in [4]. The polynomial is

$$
\frac{1}{a_{1} ! \cdots a_{k} !} \sum_{T \subset\{1, \ldots, n\}}(-1)^{|T|} f_{\lambda}(|\{1, \ldots, n\} \backslash T|) \prod_{i=1}^{k} f_{\lambda}\left(\left|A_{i} \cap T\right|\right) .
$$

Surprisingly, this polynomial turns out to be constant. As a reminder, $f_{\lambda}(n)$ is the generating function for the elements of $S_{n}$ by the number of fixed points. Thus the first few values of $f_{\lambda}$ are

$$
\begin{aligned}
& f_{\lambda}(0)=1 \\
& f_{\lambda}(1)=\lambda \\
& f_{\lambda}(2)=1+\lambda^{2} \\
& f_{\lambda}(3)=2+3 \lambda+\lambda^{3} \\
& f_{\lambda}(4)=9+8 \lambda+6 \lambda^{2}+\lambda^{4}
\end{aligned}
$$

Eriksen et al. (Section 5 of [4]) show that (4) counts the $\left(a_{1}, \ldots, a_{k}\right)$-descending derangements. They do this in two steps: they first show that (4) is equal to the number of $\left(a_{1}, \ldots, a_{k}\right)$-descending derangements when $\lambda=1$, and then they show that (4) does not depend on $\lambda$ by differentiating with respect to $\lambda$. In this section, we show combinatorially that (4) is constant.

Call a cycle of a permutation $\pi$ small if it lies entirely within one of the blocks $A_{i}$. Let $c(\pi)$ be equal to 0 if $\pi$ contains any odd-length small cycles, and let $c(\pi)$ be equal to $2^{m}$ otherwise, where $m$ is the number of small cycles in $\pi$ (which will in this case necessarily all have even length).

\section{Proposition 5.1.}

$$
\frac{1}{a_{1} ! \cdots a_{k} !} \sum_{T \subset\{1, \ldots, n\}}(-1)^{|T|} f_{\lambda}(|\{1, \ldots, n\} \backslash T|) \prod_{i=1}^{k} f_{\lambda}\left(\left|A_{i} \cap T\right|\right)=\frac{1}{a_{1} ! \cdots a_{k} !} \sum_{\pi \in S_{n}} c(\pi) .
$$


In particular, (4), which is also the left-hand side of (5), does not depend on $\lambda$, and the right-hand side of $(5)$ is the number of $\left(a_{1}, \ldots, a_{k}\right)$-descending derangements.

Proof. As noted above, Eriksen et al. have already shown that (4) counts the $\left(a_{1}, \ldots, a_{k}\right)$ descending derangements, so to prove Proposition 5.1, we only need to establish (5). that

The $\frac{1}{a_{1} ! \cdots a_{k} !}$ factor appears on both sides of (5), so we may ignore it and instead prove

$$
\sum_{T \subset\{1, \ldots, n\}}(-1)^{|T|} f_{\lambda}(|\{1, \ldots, n\} \backslash T|) \prod_{i=1}^{k} f_{\lambda}\left(\left|A_{i} \cap T\right|\right)=\sum_{\pi \in S_{n}} c(\pi) .
$$

We start by creating a multivariate version of (6). We will work in $\mathbb{C}\left[S_{n}\right]$, the group algebra of $S_{n}$. Define a function $I: 2^{S_{n}} \rightarrow \mathbb{C}\left[S_{n}\right]$ by

$$
I(T)=\sum_{\pi \in T} \pi
$$

for any $T \subset S_{n}$. Now we write down an element of $\mathbb{C}\left[S_{n}\right]$ that is similar to the sum on the left-hand-side of (6). Given a set $X$, let $\operatorname{Sym}(X)$ denote the symmetric group acting on $X$. Whenever $X \subset\{1, \ldots, n\}$, there is a natural embedding of $\operatorname{Sym}(X)$ in $S_{n}$. The desired element of $\mathbb{C}\left[S_{n}\right]$ is

$$
Q=\sum_{T \subset\{1, \ldots, n\}}(-1)^{|T|} I(\operatorname{Sym}(\{1, \ldots, n\} \backslash T)) \cdot \prod_{i=1}^{k} I\left(\operatorname{Sym}\left(A_{i} \cap T\right)\right) .
$$

The rest of the proof hinges on the following claim.

\section{Claim.}

$$
Q=\sum_{\pi \in S_{n}} c(\pi) \pi
$$

Proof of claim. Fix a permutation $\pi$ and consider the terms of $Q$ in which $\pi$ appears. That is, consider for which values of $T$ the permutation $\pi$ lies in $G_{T}:=\operatorname{Sym}(\{1, \ldots, n\} \backslash T) \times$ $\prod_{i=1}^{k} \operatorname{Sym}\left(A_{i} \cap T\right)$. The permutation $\pi$ lies in $G_{T}$ if and only if each of its cycles lies in $\{1, \ldots, n\} \backslash T$ or in $T \cap A_{i}$ for some $i$. In other words, (i) for every cycle that is not small, $\{1, \ldots, n\} \backslash T$ must contain that cycle; (ii) for every small cycle $c$, the set $\{1, \ldots, n\} \backslash T$ must either contain $c$ or be disjoint from $c$. If there is any odd-length small cycle $c$ in $\pi$ then we can pair off terms where $c \subset T$ with terms where $c \cap T=\emptyset$, and $|T|$ will have different parity in both cases, so any permutation with an odd-length small cycle cancels out of $Q$.

If $\pi$ has no odd-length small cycles, then the preceding argument shows that $|T|$ will be even whenever $\pi \in G_{T}$ (because $T$ is a union of small cycles of $\pi$ ). Therefore, $\pi$ will always appear with the same (positive) sign, and $\pi$ appears $c(\pi)$ times in this case because every small cycle of $\pi$ can either lie in $T$ or not lie in $T$. Thus the coefficient of $\pi$ in $Q$ is indeed $c(\pi)$, and the claim follows. 
Now consider the vector space homomorphism FIX : $\mathbb{C}\left[S_{n}\right] \rightarrow \mathbb{C}[\lambda]$ defined on elements of $S_{n}$ as

$$
\operatorname{FIX}(\pi)=\lambda^{|\operatorname{fix}(\pi)|}
$$

and extended by linearity to all of $\mathbb{C}\left[S_{n}\right]$. Note that $\operatorname{FIX}(Q)$ is equal to the left-hand-side of $(6)$. On the other hand, by considering (8), we see that $\operatorname{FIX}(Q)$ is equal to

$$
\sum_{\pi \in S_{n}} c(\pi) \operatorname{FIX}(\pi)
$$

However, every fixed point of $\pi$ is a small cycle of odd length. Therefore, if $\operatorname{FIX}(\pi) \neq 1$, then $c(\pi)=0$. Hence (9) simplifies to

$$
\sum_{\pi \in S_{n}} c(\pi)
$$

This is exactly the right-hand-side of (6), so the left-hand-side and right-hand-side of (6) are equal, as we wanted to show.

In the next section, we will prove directly that

$$
\frac{1}{a_{1} ! \cdots a_{k} !} \sum_{\pi \in S_{n}} c(\pi)
$$

counts the $\left(a_{1}, \ldots, a_{k}\right)$-descending derangements and also generalize this formula to count the $(A, S)$-derangements.

\section{A combinatorial sum}

We now derive a combinatorial sum for the $(A, S)$-derangements. In this section, we will make use of the maps $\Phi, \Psi$, and $\Upsilon$, which are defined at the beginning of Section 2 .

Let $c_{S}(\pi)=0$ if $\pi$ has any odd-length small cycles or small cycles in ascending blocks. Otherwise, let $c_{S}(\pi)=2^{m}$, where $m$ is the number of small cycles. The next theorem is our main result in this section.

Theorem 6.1. The number of $(A, S)$-derangements is equal to

$$
\frac{1}{a_{1} ! \cdots a_{k} !} \sum_{\pi \in S_{n}} c_{S}(\pi)
$$

We recall Corollary 2.7, which states that the $(A, S)$-derangements are in bijection with the ornaments such that (i) the number of vertices colored $i$ is equal to $a_{i}$; (ii) every cycle is aperiodic (1-repeating), with the exception of monochromatic 2-cycles from descending blocks; and (iii) there are no 1-cycles. We will call an ornament satisfying these conditions an $(A, S)$-satisfactory ornament. In view of the statement of Theorem 6.1 , we will also define an $(A, S)$-acceptable permutation as a permutation with 
- no small cycles from ascending blocks

- only even-length small cycles from descending blocks

and define an $(A, S)$-acceptable ornament as an ornament with

- no monochromatic cycles in ascending blocks

- only even-length monochromatic cycles from descending blocks

- exactly $a_{i}$ vertices colored $i$.

Thus the image of the $(A, S)$-acceptable permutations under $\Phi$ is the $(A, S)$-acceptable ornaments.

We are now ready to prove Theorem 6.1. Roughly, our strategy will be to take the $(A, S)$-acceptable permutations, map them to the $(A, S)$-acceptable ornaments with $\Phi$, map them to augmentations of $(A, S)$-satisfactory ornaments with $\Psi$, and then forget the augmentations to obtain $(A, S)$-satisfactory ornaments.

Proof of Theorem 6.1. Let $\Pi$ be the set of $(A, S)$-acceptable permutations, $\Omega$ the set of $(A, S)$-acceptable ornaments, and $\Sigma$ the set of $(A, S)$-satisfactory ornaments. Recall that we are trying to show that

$$
\frac{1}{a_{1} ! \cdots a_{k} !} \sum_{\pi \in S_{n}} c_{S}(\pi)
$$

counts the $(A, S)$-derangements. Consider the element

$$
X=\frac{1}{a_{1} ! \cdots a_{k} !} \sum_{\pi \in S_{n}} c(\pi) \pi
$$

of the group algebra $\mathbb{C}[\Pi]$. As noted earlier, $\Phi$ maps $\Pi$ into $\Omega$. Naturally extend $\Phi$ to a map from $\mathbb{C}[\Pi]$ to $\mathbb{C}[\Omega]$. Note that if $\Phi(\pi)=\Phi\left(\pi^{\prime}\right)$, then $c_{S}(\pi)=c_{S}\left(\pi^{\prime}\right)$, so we can regard $c_{S}$ as a function on ornaments by defining $c_{S}(\omega)$ to be $c_{S}\left(\Phi^{-1}(\omega)\right)$ for any $(A, S)$-acceptable ornament $\omega$.

Finally, let $N(\omega)$ denote the group of symmetries of an ornament $\omega$. So if $\omega=$ $\left\{\nu_{1}^{l_{1}}, \ldots, \nu_{m}^{l_{m}}\right\}$, and $\nu_{i}$ is $r_{i}$-repeating, then the size of $N(\omega)$ is $r_{1} l_{1} ! \cdots r_{m} l_{m} !$. In Figure 8 , we compute the number of symmetries of an ornament.
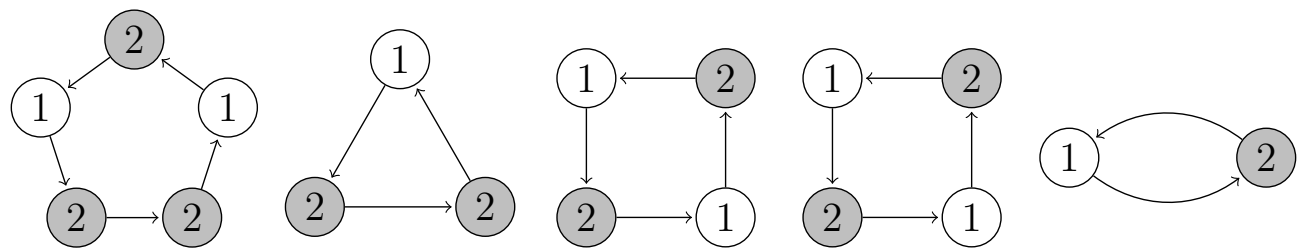

Figure 8: An $(8,10)$-compatible ornament (in fact, the same ornament as in Figure 3). White vertices are labeled 1 and grey vertices are labeled 2 . This ornament has $2^{2} \cdot 2 !=8$ symmetries, since we can permute the two squares and also rotate each of them by any multiple of 180 degrees. 


\section{Claim.}

$$
\Phi(X)=\sum_{\omega \in \Omega} \frac{c_{S}(\omega)}{|N(\omega)|} \omega
$$

Proof of claim. Given any ornament $\omega$, there are $a_{1} ! \cdots a_{k}$ ! ways to fill in the vertices of $\omega$ with the integers $\{1, \ldots, n\}$ such that the vertices labeled $i$ are assigned distinct elements of $A_{i}$. However, there is some double-counting going on, as every symmetry of $\omega$ means that two different ways of filling in the vertices of $\omega$ will actually yield the same permutation in the end. Thus we overcount by a factor of $|N(\omega)|$, hence $\frac{a_{1} ! \cdots a_{k} !}{|N(\omega)|}$ permutations map to a given ornament $\omega$ under the map $\Phi$. Since each of these permutations is assigned a weight $\frac{c_{S}(\omega)}{a_{1} ! \cdots a_{k} !}$ in the sum for $X$, the claim follows.

Now take the map $\Upsilon$ defined in Section 2. One can check that it maps $\Omega$ bijectively to the set $\Sigma$ of $(A, S)$-satisfactory ornaments. Extend $\Upsilon$ to an isomorphism from $\mathbb{C}[\Omega]$ to $\mathbb{C}[\Sigma]$.

If $\Psi(\omega)=\omega_{\lambda}^{\prime}$, then $\omega$, and hence $|N(\omega)|$, is determined by $\lambda$ and $\omega^{\prime}$. We will obtain a convenient expression for $|N(\omega)|$ in terms of $\omega^{\prime}$ and $\lambda$. Suppose that $\omega_{\lambda}^{\prime}=\left\{\nu_{1}^{\lambda_{1}}, \ldots, \nu_{m}^{\lambda_{m}}\right\}$. Also let $f(\nu)$ be equal to the $r$ for which the cycle $\nu$ is $r$-repeating. For all cases we will consider, $f(\nu)=2$ if $\nu$ is a monochromatic 2-cycle and $f(\nu)=1$ otherwise.

Claim. If $\lambda_{i}$ has $n_{i j}$ parts of size $j$ and $\left|\lambda_{i}\right|$ denotes the total number of parts of $\lambda_{i}$, then

$$
|N(\omega)|=\prod_{i}\left(f\left(\nu_{i}\right)^{\left|\lambda_{i}\right|} \prod_{j} j^{n_{i j}} n_{i j} !\right) .
$$

Proof of claim. Note that the symmetries of $\omega$ come from the internal symmetries of each cycle together with the symmetries between the cycles. In other words, every symmetry of $\omega$ permutes isomorphic cycles and also might rotate each cycle by a multiple of its period length. There are $n_{i j}$ cycles in $\omega$ that are equal to $j$ concatenated copies of $\nu_{i}$; each of these cycles has $j f\left(\nu_{i}\right)$ internal symmetries, and there are $n_{i j}$ ! ways to permute these cycles among each other, so these cycles contribute a factor of $f\left(\nu_{i}\right)^{n_{i j}} j^{n_{i j}} n_{i j}$ !. Multiplying this across all $i$ and $j$ yields (10).

In view of this, we will define $N\left(\lambda_{i}\right)=\prod_{j} j^{n_{i j}} n_{i j}$ ! and define $N(\lambda)=\prod_{i} N\left(\lambda_{i}\right)$. Thus $\frac{|N(\omega)|}{c_{S}(\omega)}=N(\lambda)$. Also, let $\lambda \vdash l$ mean that $\lambda$ is a partition of $l$. We then see that

$$
\Upsilon(\Phi(X))=\sum_{\omega^{\prime} \in \Sigma} \omega^{\prime} \sum_{\lambda} \frac{1}{N(\lambda)}=\sum_{\omega^{\prime}=\left\{\nu_{1}^{l_{1}}, \ldots, \nu_{m}^{l_{m}}\right\} \in \Sigma} \omega^{\prime} \prod_{i=1}^{m} \sum_{\lambda_{i} \vdash l_{i}} \frac{1}{N\left(\lambda_{i}\right)} .
$$

Here the sum for $\lambda$ is over all augmentations $\omega_{\lambda}^{\prime}$ of $\omega^{\prime}$, and the sum for $\lambda_{i}$ is over all partitions $\lambda_{i}$ of $l_{i}$. Our final observation is that $N\left(\lambda_{i}\right)$ is the size of the stabilizer of the conjugacy class corresponding to $\lambda_{i}$ in $S_{l_{i}}$, hence $\sum_{\lambda_{i} \vdash l_{i}} \frac{1}{N\left(\lambda_{i}\right)}=1$ by the class equation for $S_{l_{i}}$. Thus the above equation simplifies to

$$
\Upsilon(\Phi(X))=\sum_{\omega^{\prime} \in \Sigma} \omega^{\prime}
$$


which implies that $\frac{1}{a_{1} ! \cdots a_{k} !} \sum_{\pi \in S_{n}} c_{S}(\pi)$ is equal to $|\Sigma|$, which is the number of $(A, S)$ satisfactory ornaments, which by Corollary 2.7 is the number of $(A, S)$-derangements, so we are done.

If, instead of considering $(A, S)$-satisfactory ornaments, we consider ornaments that have no 1-cycles in ascending blocks and an even number of 1-cycles in each descending block, the same idea as above works. This new set of ornaments is also in bijection with the $(A, S)$-derangements, since we can replace every pair of 1-cycles from a descending block with a 2-cycle from the same block.

Running through the above argument with this new set of ornaments yields Theorem 6.2. Theorem 6.2 is interesting because instead of a sum like $\sum_{\pi \in S_{n}} c_{S}(\pi)$, we end up with the cardinality of a set because all coefficients in the sum end up being either 0 or 1. It is also more natural than Theorem 6.1 since it uses only bijections induced by the maps $\Phi$ and $\Upsilon$.

Theorem 6.2. Let $D$ be the number of $(A, S)$-derangements, and let $E$ be the number of permutations such that

- There are no small cycles in ascending blocks.

- The total length of all small cycles in each descending block is even.

Then $E=a_{1} ! \cdots a_{k} ! D$.

Proof sketch. Let $\Pi^{\prime}$ be the set of permutations with no small cycles in ascending blocks and such that the total length of all small cycles in each descending block is even. Let $\Omega^{\prime}$ be the set of all $A$-compatible ornaments with no monochromatic cycles in ascending colors and such that the total length of all monochromatic cycles in each descending color is even. Let $\Sigma^{\prime}$ be the set of $A$-good ornaments with no 1-cycles in ascending blocks and an even number of 1-cycles in each descending block. As noted in Proposition 2.6, $\Phi$ is a bijection between the $(A, S)$-derangements and $\Sigma^{\prime}$. The map $\Phi$ also sends $\Pi^{\prime}$ to $\Omega^{\prime}$, and $\Upsilon$ sends $\Omega^{\prime}$ bijectively to $\Sigma^{\prime}$. Finally, all the fibres of $\Phi$, when mapping from $\Pi^{\prime}$ to $\Omega^{\prime}$, have size $a_{1} ! \cdots a_{k}$ !. This means that the map $\Phi^{-1} \circ \Upsilon \circ \Phi$, as a map from $\Pi^{\prime}$ to the $(A, S)$-derangements, is a surjection such that each fibre has size $a_{1} ! \cdots a_{k} !$. This implies Theorem 6.2.

We also note a more direct relation between Theorem 6.1 and Theorem 6.2. We can see this by fixing a set of elements in each block that are to come from small cycles. Suppose that this set is $T$ for some block ( $T$ necessarily has even cardinality). The relevant claim is that the total number of permutations on $T$ (i.e., $|T|$ !) is equal to the sum, over all permutations with only even cycles, of 2 raised to the power of the number of cycles in the permutation. In other words, the claim is that if $S=\{1\}$ and $A=(a)$, then

$$
\sum_{\pi \in S_{n}} c_{S}(\pi)=a !
$$


If we then multiply across all blocks, we see that the sum in Theorem 6.1 is equal to $E$, which shows that Theorems 6.1 and 6.2 imply each other.

We will now prove our claim. In fact, we can prove a stronger statement.

Proposition 6.3. Let $T$ be a set whose cardinality is divisible by $m$. For a permutation $\pi$ of $T$, let $d(\pi)$ equal 0 if $\pi$ has any cycles whose length is not divisible by $m$ and let it be equal to $m^{t}$ otherwise, where $t$ is the number of cycles in $\pi$. Then

$$
\sum_{\pi \in \operatorname{Sym}(T)} d(\pi)=|T| !
$$

Proof. Let $n=|T|$. We will use the Pólya enumeration theorem [10]. Consider the set $\mathcal{F}$ of all functions from $T$ to $\{0, \ldots, m-1\}$. Then $\operatorname{Sym}(T)$ acts on $\mathcal{F}$ by pre-composition. That is, $[\pi \cdot f](t):=f(\pi(t))$ for $f \in \mathcal{F}$. We give a weight $w(f)$ to each function $f \in \mathcal{F}$ equal to the sum of the values of $f$ across $T$. In other words, $w(f)=\sum_{t \in T} f(t)$. Note that two elements in the same orbit under $\operatorname{Sym}(T)$ have the same weight, so we can define $w$ on the orbits of $\mathcal{F}$ as well.

Let $Z$ be the cycle index of $\operatorname{Sym}(T)$, that is,

$$
Z\left(x_{1}, \ldots, x_{n}\right)=\frac{1}{n !} \sum_{\pi \in \operatorname{Sym}(T)} \prod_{j=1}^{n} x_{i}^{n_{j}(\pi)}
$$

where $n_{j}(\pi)$ is equal to the number of cycles of length $j$ in $\pi$. Also, let $C(t)$ be the generating function by weight for the orbits of $\mathcal{F}$ and let $c(t)$ be the generating function by weight for the elements of $\{0, \ldots, m-1\}$. The Pólya enumeration theorem states that

$$
C(t)=Z\left(c(t), c\left(t^{2}\right), c\left(t^{3}\right), \ldots\right) .
$$

We can easily see that $c(t)=1+t+t^{2}+\ldots+t^{m-1}$. Furthermore, the orbit of a function $f \in \mathcal{F}$ is uniquely determined by $\left|f^{-1}(i)\right|$ for $i=0, \ldots, m-1$, and conversely there is a non-empty orbit of functions $f$ with $\left|f^{-1}(i)\right|=a_{i}$ for any sequence $a_{0}, \ldots, a_{m-1}$ with $a_{0}+\ldots+a_{m-1}=n$. Hence the $t^{k}$ coefficient of $C(t)$ is equal to the number of solutions, over non-negative integers, to the equations

$$
\begin{aligned}
a_{0}+a_{1}+a_{2}+\ldots+a_{m-1} & =n \\
a_{1}+2 a_{2}+\ldots+m a_{m-1} & =k .
\end{aligned}
$$

It follows that $C(t)$ is equal to the $x^{n}$ coefficient in

$$
\frac{1}{1-x} \frac{1}{1-t x} \frac{1}{1-t^{2} x} \cdots \frac{1}{1-t^{m-1} x}
$$

We will denote the $x^{n}$ coefficient of a polynomial $p$ by $\left[x^{n}\right] p$. We have then shown that

$$
\left[x^{n}\right]\left(\frac{1}{1-x} \frac{1}{1-t x} \cdots \frac{1}{1-t^{m-1} x}\right)=Z\left(1+t+\cdots+t^{m-1}, 1+t^{2}+\cdots+t^{2 m-2}, \ldots\right) .
$$


If we evaluate this at a primitive $m$ th root of unity $\zeta$, then we see that

$$
\left[x^{n}\right]\left(\frac{1}{1-x} \frac{1}{1-\zeta x} \cdots \frac{1}{1-\zeta^{m-1} x}\right)=Z(0,0, \ldots, m, 0,0, \ldots, m, \ldots),
$$

where the $m$ occurs in every $m$ th spot. But then the right-hand side is $\frac{1}{n !} \sum_{\pi \in \operatorname{Sym}(T)} d(\pi)$, and the left-hand side is $\left[x^{n}\right] \frac{1}{1-x^{m}}$. Since $n$ is divisible by $m,\left[x^{n}\right] \frac{1}{1-x^{m}}=1$. This implies that $\sum_{\pi \in \operatorname{Sym}(T)} d(\pi)=n$ !, as claimed.

\section{Conclusion and Open Problems}

In this paper we have considered the map $\Phi$ applied to $(A, S)$-permutations. It would be interesting to study $\Phi$ as a map on all permutations at once or perhaps restricted to other special classes of permutations.

One interesting class of permutations consists of permutations that are split into blocks of length $a_{1}, \ldots, a_{k}$, and such that the relative ordering of the permutation values within block $i$ agrees with some pre-determined permutation $\pi_{i}-$ so $\left(a_{1}, \ldots, a_{k}\right)$-ascending permutations would be the special case when $\pi_{i}$ is the identity permutation for all $i$. We will call the more general case an $\left(\left(a_{1}, \pi_{1}\right), \ldots,\left(a_{k}, \pi_{k}\right)\right)$-permutation. For example, a $((3,312),(2,12))$-permutation would be a permutation $\pi$ such that $\pi(2)<\pi(3)<\pi(1)$ and $\pi(4)<\pi(5)$.

Unfortunately, the map $\Phi$ is not usually injective when applied to the $\left(\left(a_{1}, \pi_{1}\right),\left(a_{2}, \pi_{2}\right)\right.$, $\left.\ldots,\left(a_{k}, \pi_{k}\right)\right)$-permutations. For instance, let $\left(\left(a_{1}, \pi_{1}\right),\left(a_{2}, \pi_{2}\right)\right)=((3,132),(1,1))$. Then $\Phi(1423)=\Phi(2431)$, as they both yield a 1-cycle with color 1 and a 3-cycle with colors $1,1,2$. If one considers large enough permutations, one can also find cases where $\Phi$ maps 3 permutations to the same ornament. In fact, it appears that $\Phi$ fails to be injective for some set of permutations $\pi_{1}, \ldots, \pi_{k}$ whenever there is some $i$ with $a_{i}>2$. (This is the smallest case that allows for a permutation $\pi_{i}$ that is neither always ascending nor always descending.) Also, the sizes of the fibres of $\Phi$ appear to vary. Thus there is no obvious structure that is preserved, at least in terms of injectivity, when we look at $\left(\left(a_{1}, \pi_{1}\right), \ldots,\left(a_{k}, \pi_{k}\right)\right)$-permutations.

It also appears that Corollary 4.1 does not hold in this case. In particular, the number of $((3,123),(3,132))$-permutations in the conjugacy class $(1,2,3)$ is not the same as the number of $((3,132),(3,123))$-permutations in the conjugacy class $(1,2,3)$. (There is a single one, $\pi=134265$ in the first case, and there are none in the second case.)

Nevertheless, one could perhaps show that when the number of inversions in each $\pi_{i}$ is bounded, so is the size of the pre-image of each ornament under $\Phi$, provided that we hold the number of blocks constant. One could also ask for ways to determine, for a given ornament, what the pre-image of $\Phi$ looks like. Perhaps there is some generalization of Lemma 2.4 or Corollary 2.5 that would hold. Since $\Phi$ is not injective in general, the conclusion would necessarily have to be weaker, but perhaps one could find a nice partial order or multi-dimensional sequence to use that would help to determine the relative order of two vertices in an ornament. 
We could also look at the map $\Psi \circ \Phi$. We could ask the same sorts of questions about this map as we asked about $\Phi$, and we will probably be more successful, since the bijection between $(A, S)$-permutations and augmentations of $A$-good ornaments seems to be the most natural of all the bijections presented in this paper. The tradeoff is that $\Psi \circ \Phi$ does not preserve cycle structure, but it should still be fairly easy to figure out the cycle structure in most cases. I believe that to understand $\Phi$, one should start by trying to understand $\Psi \circ \Phi$.

We still need a better explanation of why (4) counts the $\left(a_{1}, \ldots, a_{k}\right)$-descending derangements. Our argument right now is unsatisfying because it involves two disjoint arguments (Proposition 5.1 and Theorem 6.1) and therefore does not give a direct connection between (4) and the number of $\left(a_{1}, \ldots, a_{k}\right)$-descending derangements. It would therefore be nice to have an argument directly relating $(4)$ to the $\left(a_{1}, \ldots, a_{k}\right)$-descending derangements for all values of $\lambda$.

It would also be nice to generalize (4) to count the $(A, S)$-derangements. This is particularly tempting because (4) reduces to a special case of the equation in Theorem 6.1 , and this equation counts the $(A, S)$-derangements in general. On the other hand, the bijection from Corollary 2.7 is somewhat unnatural, so it is possible that the right way to generalize (4) would involve something closer to the bijection of Theorem 6.2.

The sum $\frac{1}{a_{1} ! \cdots a_{k} !} \sum_{\pi \in S_{n}} c_{S}(\pi)$ looks tantalizingly close to something we would get out of an application of the Pólya enumeration theorem. We in fact proved Proposition 6.3, a result closely related to this sum, using the Pólya enumeration theorem. So it would be interesting to see if we could prove that $\frac{a_{1} ! \cdots a_{k} !}{\sum}{ }_{\pi \in S_{n}}$ enumerates the $(A, S)$-derangements using Pólya theory, or, even better, drawing a connection between that sum and the polynomial sum in [4].

Another question is whether we can obtain a recursion, similar to that for $f_{j}$, for the number of derangements with exactly a given descent set. This is different from looking at $(A, S)$-permutations because with $(A, S)$-permutations there are certain points (between the blocks) when a permutation can either ascend or descend, and so the descent set is never specified completely. A starting point would be to find an elegant recursion for the permutations with a given descent set. We can already count the permutations with a given descent set using inclusion-exclusion (see for example Theorem 1.4 of [1]), but a recursive enumeration might be more flexible and thus allow us to incorporate the constraint that the permutations also be derangements more easily.

We could also ask for the asymptotic density of the $(A, S)$-derangements in the $(A, S)$ permutations. Is it, as in the case of all derangements, roughly $\frac{1}{e}$ ? In Section 7 of [4], Eriksen et al. show that being a derangement and being an $\left(a_{1}, \ldots, a_{k}\right)$-descending permutation are positively correlated events, but it is possible that they are not strongly correlated enough to affect the asymptotics.

There are a couple ways to get a notion of asymptotic density. We could first of all fix $S$ and demand that each of the block sizes gets large. In other words, we could ask if there exists an $\alpha$ such that for any sequence of $k$-tuples of positive integers $\left(a_{1 j}, \ldots, a_{k j}\right)$ such that $\min _{i=1}^{k} a_{i j}$ goes to infinity with $j$, the density of the $\left(a_{1 j}, \ldots, a_{k j}, S\right)$-derangements in the $\left(a_{1 j}, \ldots, a_{k j}, S\right)$-permutations approaches $\alpha$. We could also fix $S$ and all of $a_{1}, \ldots, a_{k}$ 
and look at the $\left(c a_{1}, \ldots, c a_{k}, S\right)$-derangements for $c=1,2, \ldots$, and then ask the same question. Even better would be to actually compute $\alpha$ (which would depend on $S$ and, in the latter formulation, also on $a_{1}, \ldots, a_{k}$ ).

Another direction for further research is to find a polynomial-time algorithm to count the $(A, S)$-derangements. All current algorithms take time exponential in the number of blocks. One difficulty is that even a very efficient recursion will probably have $k$ variables and so even a dynamic programming approach will take exponential time.

A final direction of further inquiry involves a simpler proof of one of our results. In the proof of Corollary 4.2, we used the fact that there are $\left(\begin{array}{c}n \\ a_{1}, \ldots . a_{k}\end{array}\right)$ A-good ornaments. The enumeration of $A$-good ornaments required Theorem 2.2. However, the simplicity of both the question "How many $A$-good ornaments are there?" and its answer suggests

that there should be a more direct proof that there are $\left(\begin{array}{c}n \\ a_{1}, \ldots, a_{k}\end{array}\right)$ A-good ornaments. Can we find such a proof?

\section{Acknowledgements}

This research was supervised by Joe Gallian at the University of Minnesota Duluth, supported by the National Science Foundation and the Department of Defense (grant number DMS 0754106) and the National Security Agency (grant number H98230-06-10013).

In addition to Joe Gallian, the author thanks Reid Barton, Paul Christiano, Ricky Liu, Aaron Pixton, Allan Steinhardt, and Phil Matchett Wood for help with the paper itself. He particularly thanks Ricky Liu and Reid Barton for pointing out the idea of templates for use in proving Theorem 2.2. He also thanks Richard Peng for pointing out a correspondence between the Gessel-Reutenauer bijection and the Burrows-Wheeler transform, as well as Geir Helleloid, Adam Hesterburg, Nathan Kaplan, Nathan Pflueger, and Yufei Zhao for helpful conversations. Finally, he thanks the anonymous referee for correcting various errors and making helpful suggestions on the organization of the paper.

\section{Glossary}

This section is intended for reference only. All necessary definitions will also be given either in the introduction or the body of the paper.

- ascent: an index $i$ of a permutation $\pi$ on $\{1, \ldots, n\}$ such that $\pi(i)<\pi(i+1)$

- descent: an index $i$ of a permutation $\pi$ on $\{1, \ldots, n\}$ such that $\pi(i)>\pi(i+1)$

- excedance: an index $i$ of a permutation $\pi$ on $\{1, \ldots, n\}$ such that $\pi(i)>i$

- deficiency: an index $i$ of a permutation $\pi$ on $\{1, \ldots, n\}$ such that $\pi(i)<i$

- fixed point: an index $i$ of a permutation $\pi$ on $\{1, \ldots, n\}$ such that $\pi(i)=i$ 
- derangement: a permutation with no fixed points

- $\left(a_{1}, \ldots, a_{k}\right)$-ascending permutation: a permutation that ascends within consecutive blocks of lengths $a_{1}, \ldots, a_{k}$; in other words, its descent set is contained in $\left\{a_{1}, a_{1}+a_{2}\right.$, $\left.\ldots, a_{1}+\cdots+a_{k-1}\right\}$. The blocks are referred to as $A_{1}, \ldots, A_{k}$, so, for example, $A_{1}=\left\{1, \ldots, a_{1}\right\}$.

- $\left(a_{1}, \ldots, a_{k}\right)$-descending permutation: a permutation that descends in consecutive blocks of lengths $a_{1}, \ldots, a_{k}$. Once again the blocks are referred to as $A_{1}, \ldots, A_{k}$.

- $\left(a_{1}, \ldots, a_{k}, S\right)$-permutation or $(A, S)$-permutation (in this case $A=\left(a_{1}, \ldots, a_{k}\right)$ implicitly): a permutation that, when split into blocks $A_{1}, \ldots, A_{k}$ of lengths $a_{1}, \ldots, a_{k}$, descends in the blocks $A_{i}$ for $i \in S$ and ascends in the other blocks.

- necklace: a directed cycle whose vertices are either colored or labeled; Figure 1 has an example of a necklace. Necklaces are also sometimes called cycles.

- fundamental period: the smallest contiguous subsequence $P$ of a necklace such that the necklace is $r$ copies of $P$ for some $r$.

- $r$-repeating: a necklace is $r$-repeating for the value of $r$ in the preceding definition

- ornament: a multiset of necklaces; Figure 1 has an example of an ornament.

- $\left(a_{1}, \ldots, a_{k}\right)$-compatible ornament or $A$-compatible ornament: an ornament whose vertices are colored by the integers $\{1, \ldots, k\}$ such that $a_{i}$ vertices are colored by $i$. In this case we are either implicitly or explicitly considering $(A, S)$-permutations as well, so there is an associated subset $S$ of $\{1, \ldots, k\}$.

- ascending color: a color that does not lie in the set $S$ in the above definition

- descending color: a color that does lie in the set $S$

- $\left(a_{1}, \ldots, a_{k}\right)$-good ornament or $A$-good ornament: an $A$-compatible ornament such that every necklace is 1-repeating

- $\Phi$ : a map from permutations to $A$-compatible ornaments; it takes a permutation, writes it as a product of disjoint cycles, and replaces each element of each cycle by the block it belongs to

- I: a map from $A$-compatible ornaments to augmentations of $A$-good ornaments; for each cycle of an ornament $\omega$ that is $r$-repeating, replace it with $r$ copies of its fundamental period. This gives us an $A$-good ornament $\omega^{\prime}$. If $\rho$ is a necklace of $\omega^{\prime}$, and $n_{r}$ necklaces in $\omega$ have fundamental period $\rho$ and are $r$-repeating, then the partition augmenting $\rho$ has $n_{r}$ parts of size $r$.

- $\Upsilon$ : a map from $A$-compatible ornaments to $A$-good ornaments. It is obtained by applying $\Psi$, then forgetting the augmentation to end up with just an ornament. 
- walk: the sequence $W(v)$ defined in Lemma 2.4

- signed walk: the sequence $A(v)$ defined in Corollary 2.5

- packet: Given a vertex $v$ of an ornament, the packet of $v$ is the set $P(v):=\left\{v^{\prime} \mid\right.$ $\left.A\left(v^{\prime}\right)=A(v)\right\}$; if $v$ is in a 1-repeating necklace $\nu$, we can think of $P(v)$ as the set of all vertices in necklaces isomorphic to $\nu$ in the position corresponding to $v$.

- $\left(a_{1}, \ldots, a_{k}, S\right)$-acceptable permutation or $(A, S)$-acceptable permutation: a permutation with no small cycles from ascending blocks and only even-length small cycles from descending blocks

- $\left(a_{1}, \ldots, a_{k}, S\right)$-acceptable ornament or $(A, S)$-acceptable ornament: an $\left(a_{1}, \ldots, a_{k}\right)$ compatible ornament with no monochromatic small cycles from ascending blocks and only even-length monochromatic small cycles from descending blocks

- $\left(a_{1}, \ldots, a_{k}, S\right)$-satisfactory ornament or $(A, S)$-satisfactory ornament: an ornament that is $\left(a_{1}, \ldots, a_{k}\right)$-compatible, that has no 1-cycles, and such that all cycles are aperiodic, except possibly for monochromatic 2-cycles

\section{References}

[1] Mikós Bóna. Combinatorics of Permutations. Discrete Mathematics and its Applications. Chapman and Hall/CRC, 2004.

[2] Maxime Crochemore, Jacques Désarménien, and Dominique Perrin. A note on the burrows-wheeler transformation. arXiv, abs/cs/0502073v1, 2005.

[3] Sergei Elizalde. Descent sets on cyclic permutations. arXiv, abs/0906.2795v1, 2009.

[4] Niklas Eriksen, Ragnar Freij, and Johan Wästlund. Enumeration of derangements with descents in prescribed positions. Electron. J. Combin., 16:R32, 2009.

[5] Ira M. Gessel and Christophe Reutenauer. Counting permutations with given cycle structure and descent set. J. Combin. Theory Ser. A, 64(2):189-215, 1993.

[6] Guo-Niu Han and Guoce Xin. Permutations with extremal number of fixed points. arXiv, abs/0706.1738v2, 2007.

[7] M. Lothaire. Algebraic Combinatorics on Words (online version). Cambridge University Press, 2002.

[8] Victor Reiner. Signed permutation statistics and cycle type. European J. Combin., 14:569-579, 1993.

[9] Richard Stanley. Alternating permutations and symmetric functions. arXiv, math/0603520v3, 2006.

[10] Richard Stanley. Lectures in algebraic combinatorics. http: //www-math. mit. edu/ rstan/algcomb. pdf, section 7:56-81, 2009.

[11] Philip Matchett Wood and Doron Zeilberger. A translation method for finding combinatorial bijections. Ann. Combin. (to appear). 